\title{
Relaxed Viscosity Approximation Methods with Regularization for Constrained Minimization Problems
}

\author{
Lu-Chuan Ceng, ${ }^{1}$ Hong-Kun $\mathrm{Xu},{ }^{2,3}$ and Ching-Feng Wen ${ }^{4}$ \\ ${ }^{1}$ Department of Mathematics, Shanghai Normal University, Scientific Computing Key Laboratory of Shanghai Universities, \\ Shanghai 200234, China \\ ${ }^{2}$ Department of Applied Mathematics, National Sun Yat-sen University, Kaohsiung 80424, Taiwan \\ ${ }^{3}$ Department of Mathematics, King Abdulaziz University, P.O. Box 80203, Jeddah 21589, Saudi Arabia \\ ${ }^{4}$ Center for Fundamental Science, Kaohsiung Medical University, Kaohsiung 807, Taiwan
}

Correspondence should be addressed to Ching-Feng Wen; cfwen@kmu.edu.tw

Received 13 January 2013; Accepted 26 March 2013

Academic Editor: Luigi Muglia

Copyright (c) $2013 \mathrm{Lu}$-Chuan Ceng et al. This is an open access article distributed under the Creative Commons Attribution License, which permits unrestricted use, distribution, and reproduction in any medium, provided the original work is properly cited.

We introduce a new relaxed viscosity approximation method with regularization and prove the strong convergence of the method to a common fixed point of finitely many nonexpansive mappings and a strict pseudocontraction that also solves a convex minimization problem and a suitable equilibrium problem.

\section{Introduction}

Let $H$ be a real Hilbert space with inner product $\langle\cdot, \cdot\rangle$ and norm $\|\cdot\|, C$ a nonempty closed convex subset of $H$, and $P_{C}$ the metric projection of $H$, onto $C$. Let $T: C \rightarrow C$ be selfmapping on $C$. We denote by $\operatorname{Fix}(T)$ the set of fixed points of $T$ and by $\mathbf{R}$ the set of all real numbers. A mapping $T: C \rightarrow C$ is called $\zeta$-strictly pseudocontractive if there exists a constant $\zeta \in[0,1)$ such that

$$
\begin{array}{r}
\|T x-T y\|^{2} \leq\|x-y\|^{2}+\zeta\|(I-T) x-(I-T) y\|^{2}, \\
\forall x, y \in C .
\end{array}
$$

In particular, if $\zeta=0$, then $T$ is called a nonexpansive mapping. A mapping $A: C \rightarrow H$ is called $\alpha$-inverse strongly monotone, if there exists a constant $\alpha>0$ such that

$$
\langle A x-A y, x-y\rangle \geq \alpha\|x-y\|^{2}, \quad \forall x, y \in C .
$$

Let $f: C \rightarrow \mathbf{R}$ be a convex and a continuous Fréchet differentiable functional. Consider the minimization problem (MP) of minimizing $f$ over the constraint set $C$

$$
\min _{x \in C} f(x)
$$

where we assume the existence of minimizers. We denote by $\Gamma$ the set of minimizers of (3). The gradient-projection algorithm (GPA) generates a sequence $\left\{x_{n}\right\}$ determined by the gradient $\nabla f$ and the metric projection $P_{C}$ as follows:

$$
x_{n+1}:=P_{C}\left(x_{n}-\lambda \nabla f\left(x_{n}\right)\right), \quad \forall n \geq 0,
$$

or more generally,

$$
x_{n+1}:=P_{C}\left(x_{n}-\lambda_{n} \nabla f\left(x_{n}\right)\right), \quad \forall n \geq 0,
$$

where, in both (4) and (5), the initial guess $x_{0}$ is taken from $C$ arbitrarily, the parameters $\lambda$ or $\lambda_{n}$ are positive real numbers. The convergence of algorithms (4) and (5) depends on the behavior of the gradient $\nabla f$. As a matter of fact, it is known that if $\nabla f$ is strongly monotone and Lipschitz continuous, then, for $0<\lambda<2 \alpha / L^{2}$, the operator

$$
S:=P_{C}(I-\lambda \nabla f)
$$

is a contraction. Hence, the sequence $\left\{x_{n}\right\}$ defined by the GPA (4) converges in norm to the unique solution of (3). More generally, if the sequence $\left\{\lambda_{n}\right\}$ is chosen to satisfy the property

$$
0<\liminf _{n \rightarrow \infty} \lambda_{n} \leq \limsup _{n \rightarrow \infty} \lambda_{n}<\frac{2 \alpha}{L^{2}},
$$


then the sequence $\left\{x_{n}\right\}$ defined by the GPA (5) converges in norm to the unique minimizer of (3). If the gradient $\nabla f$ is only assumed to be a Lipschitz continuous, then $\left\{x_{n}\right\}$ can only be weakly convergent if $H$ is infinite dimensional. A counterexample is given by $\mathrm{Xu}$ in [1].

Since the Lipschitz continuity of the gradient $\nabla f$ implies that it is inverse strongly monotone (ism), it can be expressed as a proper convex combination of the identity mapping and a nonexpansive mapping. Consequently, the GPA can be rewritten as the composite of a projectionand an averaged mapping which is again an averaged mapping. This shows that averaged mappings play an important role in the GPA. Very recently, $\mathrm{Xu}$ [1] used averaged mappings to study the convergence analysis of the GPA which is an operator-oriented approach.

We observe that the regularization, in particular, the traditional Tikhonov regularization, is usually used to solve ill-posed optimization problems. Consider the following regularized minimization problem:

$$
\min _{x \in C} f_{\alpha}(x):=f(x)+\frac{\alpha}{2}\|x\|^{2},
$$

where $\alpha>0$ is the regularization parameter and again $f$ is convex with an $L$-Lipschitz continuous gradient $\nabla f$.

The advantage of a regularization method is that it is possible to get strong convergence to the minimum-norm solution of the optimization problem under investigation. The disadvantage is however its implicity, and hence explicit iterative methods seem more attractive. See, for example, [1].

Given a mapping $A: C \rightarrow H$, the classical variational inequality problem (VIP) is to find $x^{*} \in C$ such that

$$
\left\langle A x^{*}, x-x^{*}\right\rangle \geq 0, \quad \forall x \in C .
$$

The solution set of VIP $(9)$ is denoted by $\operatorname{VI}(C, A)$. It is well known that $x^{*} \in \operatorname{VI}(C, A)$ if and only if $x^{*}=P_{C}\left(x^{*}-\lambda A x^{*}\right)$ for some $\lambda>0$. The variational inequality was first discussed by Lions [2] and now is well known. The variational inequality theory has been studied quite extensively and has emerged as an important tool in the study of a wide class of obstacle, unilateral, free, moving, and equilibrium problems arising in several branches of pure and applied sciences in a unified and general framework. See, for example, [3-10] and the references therein.

In this paper, we study the following equilibrium problem (EP) which is to find $x^{*} \in C$ such that

$$
F\left(x^{*}, y\right)+h\left(x^{*}, y\right) \geq 0, \quad \forall y \in C .
$$

The solution set of EP (10) is denoted by $\operatorname{EP}(F, h)$. We will introduce and consider a relaxed viscosity iterative scheme with regularization for finding a common element of the solution set $\Gamma$ of the minimization problem (3), the solution set $\operatorname{EP}(F, h)$ of the equilibrium problem (10), and the common fixed point set $\operatorname{Fix}(T) \cap\left(\bigcap_{i} \operatorname{Fix}\left(S_{i}\right)\right)$ of finitely many nonexpansive mappings $S_{i}: C \rightarrow C, i=1, \ldots, N$, and a strictly pseudocontractive mapping $T$ in the setting of the infinitedimensional Hilbert space. We will prove that this iterative scheme converges strongly to a common fixed point of the mappings $T, S_{i}: C \rightarrow C, i=1, \ldots, N$, which is both a minimizer of MP (3) and an equilibrium point of EP (10).

\section{Preliminaries}

Let $H$ be a real Hilbert space whose inner product and norm are denoted by $\langle\cdot, \cdot\rangle$ and $\|\cdot\|$, respectively. Let $K$ be a nonempty closed convex subset of $H$. We write $x_{n} \rightarrow x$ to indicate that the sequence $\left\{x_{n}\right\}$ converges weakly to $x$ and $x_{n} \rightarrow x$ to indicate that the sequence $\left\{x_{n}\right\}$ converges strongly to $x$. Moreover, we use $\omega_{w}\left(x_{n}\right)$ to denote the weak $\omega$-limit set of the sequence $\left\{x_{n}\right\}$ and $\omega_{s}\left(x_{n}\right)$ to denote the strong $\omega$-limit set of the sequence $\left\{x_{n}\right\}$; that is,

$$
\begin{aligned}
& \omega_{w}\left(x_{n}\right):=\left\{x \in H: x_{n_{i}} \rightarrow x\right. \\
& \left.\quad \text { for some subsequence }\left\{x_{n_{i}}\right\} \text { of }\left\{x_{n}\right\}\right\}, \\
& \omega_{s}\left(x_{n}\right):=\left\{x \in H: x_{n_{i}} \longrightarrow x\right. \\
& \left.\quad \text { for some subsequence }\left\{x_{n_{i}}\right\} \text { of }\left\{x_{n}\right\}\right\} .
\end{aligned}
$$

The metric (or nearest point) projection from $H$ onto $K$ is the mapping $P_{K}: H \rightarrow K$ which assigns to each point $x \in H$ the unique point $P_{K} x \in K$ satisfying the property

$$
\left\|x-P_{K} x\right\|=\inf _{y \in K}\|x-y\|=: d(x, K) .
$$

Some important properties of projections are gathered in the following.

Proposition 1. For given $x \in H$ and $z \in K$

(i) $z=P_{K} x \Leftrightarrow\langle x-z, y-z\rangle \leq 0$, for all $y \in K$;

(ii) $z=P_{K} x \Leftrightarrow\|x-z\|^{2} \leq\|x-y\|^{2}-\|y-z\|^{2}$, for all $y \in K$;

(iii) $\left\langle P_{K} x-P_{K} y, x-y\right\rangle \geq\left\|P_{K} x-P_{K} y\right\|^{2}$, for all $y \in$ $H$, which hence implies that $P_{K}$ is nonexpansive and monotone.

Definition 2. A mapping $T: H \rightarrow H$ is said to be

(a) nonexpansive if

$$
\|T x-T y\| \leq\|x-y\|, \quad \forall x, y \in H ;
$$

(b) firmly nonexpansive if $2 \mathrm{~T}-\mathrm{I}$ is nonexpansive, or equivalently,

$$
\langle x-y, T x-T y\rangle \geq\|T x-T y\|^{2}, \quad \forall x, y \in H ;
$$

alternatively, $T$ is firmly nonexpansive if and only if $T$ can be expressed as

$$
T=\frac{1}{2}(I+S),
$$

where $S: H \rightarrow H$ is nonexpansive; projections are firmly nonexpansive.

Definition 3. Let $T$ be a nonlinear operator with domain $D(T) \subseteq H$ and range $R(T) \subseteq H$. 
(a) $T$ is said to be monotone if

$$
\langle x-y, T x-T y\rangle \geq 0, \quad \forall x, y \in D(T) .
$$

(b) Given a number $\beta>0, T$ is said to be $\beta$ strongly monotone if

$$
\langle x-y, T x-T y\rangle \geq \beta\|x-y\|^{2}, \quad \forall x, y \in D(T) .
$$

(c) Given a number $v>0, T$ is said to be $\nu$-inverse strongly monotone $(\nu$-ism) if

$$
\langle x-y, T x-T y\rangle \geq v\|T x-T y\|^{2}, \quad \forall x, y \in D(T) .
$$

It can be easily seen that if $T$ is nonexpansive, then $I-T$ is monotone. It is also easy to see that a projection $P_{K}$ is 1-ism. Inverse strongly monotone (also referred to as cocoercive) operators have been applied widely in solving practical problems in various fields.

Definition 4. A mapping $T: H \rightarrow H$ is said to be an averaged mapping if it can be written as the average of the identity $I$ and a nonexpansive mapping; that is,

$$
T \equiv(1-\alpha) I+\alpha S
$$

where $\alpha \in(0,1)$ and $S: H \rightarrow H$ is nonexpansive. More precisely, when the last equality holds, we say that $T$ is $\alpha$ averaged. Thus, firmly nonexpansive mappings (in particular, projections) are (1/2)-averaged maps.

Proposition 5 (see [11]). Let $T: H \rightarrow H$ be a given mapping.

(i) $T$ is nonexpansive if and only if the complement $I-T$ is $(1 / 2)-i s m$.

(ii) If $T$ is $\nu$-ism, then for $\gamma>0, \gamma T$ is $(\nu / \gamma)$-ism.

(iii) $T$ is averaged if and only if the complement $I-T$ is $\nu$ ism for some $v>1 / 2$. Indeed, for $\alpha \in(0,1), T$ is $\alpha$ averaged if and only if $I-T$ is $(1 / 2 \alpha)-i s m$.

Proposition 6 (see [11]). Let $S, T, V: H \rightarrow H$ be given operators.

(i) If $T=(1-\alpha) S+\alpha V$ for some $\alpha \in(0,1)$ and if $S$ is averaged and $V$ is nonexpansive, then $T$ is averaged.

(ii) $T$ is firmly nonexpansive if and only if the complement $I-T$ is firmly nonexpansive.

(iii) If $T=(1-\alpha) S+\alpha V$ for some $\alpha \in(0,1)$ and if $S$ is firmly nonexpansive and $V$ is nonexpansive, then $T$ is averaged.

(iv) The composite of finitely many averaged mappings is averaged. That is, if each of the mappings $\left\{T_{i}\right\}_{i=1}^{N}$ is averaged, then so is the composite $T_{1} \cdots T_{N}$. In particular, if $T_{1}$ is $\alpha_{1}$-averaged and $T_{2}$ is $\alpha_{2}$-averaged, where $\alpha_{1}, \alpha_{2} \in(0,1)$, then the composite $T_{1} T_{2}$ is $\alpha$ averaged, where $\alpha=\alpha_{1}+\alpha_{2}-\alpha_{1} \alpha_{2}$.

(v) If the mappings $\left\{T_{i}\right\}_{i=1}^{N}$ are averaged and have a common fixed point, then

$$
\bigcap_{i=1}^{N} \operatorname{Fix}\left(T_{i}\right)=\operatorname{Fix}\left(T_{1} \cdots T_{N}\right) .
$$

The notation $\operatorname{Fix}(T)$ denotes the set of all fixed points of the mapping $T$, that is, $\operatorname{Fix}(T)=\{x \in H: T x=x\}$.

It is clear that, in a real Hilbert space $H, T: C \rightarrow C$ is $\zeta$-strictly pseudocontractive if and only if there holds the following inequality:

$$
\begin{aligned}
\langle T x-T y, x-y\rangle \leq & \|x-y\|^{2}-\frac{1-\zeta}{2} \\
& \times\|(I-T) x-(I-T) y\|^{2}, \quad \forall x, y \in C .
\end{aligned}
$$

This immediately implies that if $T$ is a $\zeta$-strictly pseudocontractive mapping, then $I-T$ is $((1-\zeta) / 2)$-inverse strongly monotone; for further detail, we refer to [12] and the references therein. It is well known that the class of strict pseudocontractions strictly includes the class of nonexpansive mappings.

Lemma 7 (see [12, Proposition 2.1]). Let $C$ be a nonempty closed convex subset of a real Hilbert space $H$ and $T: C \rightarrow C$ be a mapping.

(i) If $T$ is a $\zeta$-strictly pseudocontractive mapping, then $T$ satisfies the Lipschitz condition where

$$
\|T x-T y\| \leq \frac{1+\zeta}{1-\zeta}\|x-y\|, \quad \forall x, y \in C .
$$

(ii) If $T$ is a $\zeta$-strictly pseudocontractive mapping, then the mapping $I-T$ is semiclosed at 0 ; that is, if $\left\{x_{n}\right\}$ is a sequence in $C$ such that $x_{n} \rightarrow \tilde{x}$ weakly and (I$T) x_{n} \rightarrow 0$ strongly, then $(I-T) \tilde{x}=0$.

(iii) If $T$ is a $\zeta$-(quasi-)strict pseudocontraction, then the fixed point set $\operatorname{Fix}(T)$ of $T$ is closed and convex so that the projection $P_{\mathrm{Fix}(T)}$ is well defined.

The following lemma is an immediate consequence of an inner product.

Lemma 8. In a real Hilbert space $H$, there holds the following inequality:

$$
\|x+y\|^{2} \leq\|x\|^{2}+2\langle y, x+y\rangle, \quad \forall x, y \in H .
$$

The following elementary result on real sequences is quite well known.

Lemma 9 (see [13]). Let $\left\{a_{n}\right\}$ be a sequence of nonnegative real numbers satisfying the property

$$
a_{n+1} \leq\left(1-s_{n}\right) a_{n}+s_{n} t_{n}+\epsilon_{n}, \quad \forall n \geq 0,
$$

where $\left\{s_{n}\right\} \subset(0,1]$ and $\left\{t_{n}\right\}$ are the real sequences such that

(i) $\sum_{n=0}^{\infty} s_{n}=\infty$;

(ii) either limsup $\sup _{n \rightarrow \infty} t_{n} \leq 0$ or $\sum_{n=0}^{\infty} s_{n}\left|t_{n}\right|<\infty$;

(iii) $\sum_{n=0}^{\infty} \epsilon_{n}<\infty$ where $\epsilon_{n} \geq 0$, for all $n \geq 0$.

Then, $\lim _{n \rightarrow \infty} a_{n}=0$. 
Lemma 10 (see [14]). Let $C$ be a nonempty closed convex subset of a real Hilbert space $H$. Let $T: C \rightarrow C$ be a $\zeta$-strictly pseudocontractive mapping. Let $\gamma$ and $\delta$ be two nonnegative real numbers such that $(\gamma+\delta) \zeta \leq \gamma$. Then,

$$
\|\gamma(x-y)+\delta(T x-T y)\| \leq(\gamma+\delta)\|x-y\|, \quad \forall x, y \in C .
$$

The following lemma appears implicitly in the paper of Reinermann [15].

Lemma 11 (see [15]). Let $H$ be a real Hilbert space. Then, for all $x, y \in H$ and $\lambda \in[0,1]$,

$$
\begin{aligned}
& \|\lambda x+(1-\lambda) y\|^{2} \\
& \quad=\lambda\|x\|^{2}+(1-\lambda)\|y\|^{2}-\lambda(1-\lambda)\|x-y\|^{2} .
\end{aligned}
$$

Lemma 12 (see [16]). Let $C$ be a nonempty closed convex subset of a real Hilbert space $H$. Let $F: C \times C \rightarrow \mathbf{R}$ be a bifunction such that

(f1) $F(x, x)=0$ for all $x \in C$;

(f2) $F$ is monotone and upper hemicontinuous in the first variable

(f3) $F$ is lower semicontinuous and convex in the second variable.

Let $h: C \times C \rightarrow \mathbf{R}$ be a bifunction such that

(h1) $h(x, x)=0$ for all $x \in C$;

(h2) $h$ is monotone and weakly upper semicontinuous in the first variable;

(h3) $h$ is convex in the second variable.

Moreover, let one suppose that

(H) for fixed $r>0$ and $x \in C$, there exists a bounded $K \subset C$ and $\hat{x} \in K$ such that for all $z \in C \backslash K,-F(\widehat{x}, z)+$ $h(z, \widehat{x})+(1 / r)\langle\widehat{x}-z, z-x\rangle<0$.

For $r>0$ and $x \in H$, let $T_{r}: H \rightarrow 2^{C}$ be a mapping defined by

$$
\begin{aligned}
T_{r} x & \\
= & \{z \in C: F(z, y)+h(z, y) \\
& \left.+\frac{1}{r}\langle y-z, z-x\rangle \geq 0, \forall y \in C\right\}
\end{aligned}
$$

called the resolvent of $F$ and $h$. Then,

(1) $T_{r} x \neq \emptyset$;

(2) $T_{r} x$ is a singleton;

(3) $T_{r}$ is firmly nonexpansive;

(4) $\operatorname{EP}(F, h)=\operatorname{Fix}\left(T_{r}\right)$ and it is closed and convex.

Lemma 13 (see [16]). Let one suppose that $(f 1)-(f 3),(h 1)-(h 3)$ and $(H)$ hold. Let $x, y \in H, r_{1}, r_{2}>0$. Then,

$$
\left\|T_{r_{2}} y-T_{r_{1}} x\right\| \leq\|y-x\|+\left|\frac{r_{2}-r_{1}}{r_{2}}\right|\left\|T_{r_{2}} y-y\right\| .
$$

Lemma 14 (see [17]). Suppose that the hypotheses of Lemma 12 are satisfied. Let $\left\{r_{n}\right\}$ be a sequence in $(0, \infty)$ with $\liminf _{n \rightarrow \infty} r_{n}>0$. Suppose that $\left\{x_{n}\right\}$ is a bounded sequence. Then, the following statements are equivalent and true.

(a) if $\left\|x_{n}-T_{r_{n}} x_{n}\right\| \rightarrow 0$ as $n \rightarrow \infty$, each weak cluster point of $\left\{x_{n}\right\}$ satisfies the problem:

$$
F(x, y)+h(x, y) \geq 0, \quad \forall y \in C,
$$

that is, $\omega_{w}\left(x_{n}\right) \subseteq \mathrm{EP}(F, h)$.

(b) The demiclosedness principle holds in the sense that, if $x_{n} \rightarrow x^{*}$ and $\left\|x_{n}-T_{r_{n}} x_{n}\right\| \rightarrow 0$ as $n \rightarrow \infty$, then $\left(I-T_{r_{k}}\right) x^{*}=0$ for all $k \geq 1$.

\section{Main Results}

We now propose the following relaxed viscosity iterative scheme with regularization:

$$
\begin{gathered}
F\left(u_{n}, y\right)+h\left(u_{n}, y\right)+\frac{1}{r_{n}}\left\langle y-u_{n}, u_{n}-x_{n}\right\rangle \geq 0, \\
\forall y \in C, \\
y_{n, 1}=\beta_{n, 1} S_{1} u_{n}+\left(1-\beta_{n, 1}\right) u_{n}, \\
y_{n, i}=\beta_{n, i} S_{i} u_{n}+\left(1-\beta_{n, i}\right) y_{n, i-1}, \quad i=2, \ldots, N, \\
y_{n}=\beta_{n} Q y_{n, N}+\left(1-\beta_{n}\right) P_{C}\left(y_{n, N}-\lambda \nabla f_{\alpha_{n}}\left(y_{n, N}\right)\right), \\
x_{n+1}=\sigma_{n} y_{n}+\gamma_{n} P_{C}\left(y_{n}-\lambda \nabla f_{\alpha_{n}}\left(y_{n}\right)\right) \\
+\delta_{n} T P_{C}\left(y_{n}-\lambda \nabla f_{\alpha_{n}}\left(y_{n}\right)\right),
\end{gathered}
$$

for all $n \geq 0$, where the mapping $Q: C \rightarrow C$ is a $\rho$ contraction; the mapping $T: C \rightarrow C$ is a $\zeta$-strict pseudocontraction; $S_{i}: C \rightarrow C$ is a nonexpansive mapping for each $i=$ $1, \ldots, N ; \nabla f: C \rightarrow H$ satisfies the Lipschitz condition (10) with $0<\lambda<(2 / L) ; F, h: C \times C \rightarrow \mathbf{R}$ are two bifunctions satisfying the hypotheses of Lemma $12 ;\left\{\alpha_{n}\right\}$ is a sequence in $(0, \infty)$ with $\sum_{n=0}^{\infty} \alpha_{n}<\infty ;\left\{\beta_{n}\right\},\left\{\sigma_{n}\right\}$ are sequences in $(0,1)$ with $0<\liminf _{n \rightarrow \infty} \sigma_{n} \leq \limsup _{n \rightarrow \infty} \sigma_{n}<1 ;\left\{\gamma_{n}\right\},\left\{\delta_{n}\right\}$ are sequences in $[0,1]$ with $\sigma_{n}+\gamma_{n}+\delta_{n}=1$, for all $n \geq 0 ;\left\{\beta_{n, i}\right\}_{i=1}^{N}$ are sequences in $(0,1)$ and $\left(\gamma_{n}+\delta_{n}\right) \zeta \leq \gamma_{n}$, for all $n \geq 0$; $\left\{r_{n}\right\}$ is a sequence in $(0, \infty)$ with $\liminf _{n \rightarrow \infty} r_{n}>0$ and $\liminf _{n \rightarrow \infty} \delta_{n}>0$.

Before stating and proving the main convergence results, we first establish the following lemmas.

Lemma 15. Let one suppose that $\Omega=\operatorname{Fix}(T) \cap\left(\bigcap_{i} \operatorname{Fix}\left(S_{i}\right)\right) \cap$ $\operatorname{EP}(F, h) \cap \Gamma \neq \emptyset$. Then, the sequences $\left\{x_{n}\right\},\left\{y_{n}\right\},\left\{y_{n, i}\right\}$ for all $i$, and $\left\{u_{n}\right\}$ are bounded.

Proof. First of all, we can show as in [18] that $P_{C}\left(I-\lambda \nabla f_{\alpha}\right)$ is nonexpansive for $\lambda \in(0,2 /(\alpha+L))$, and $P_{C}\left(I-\lambda \nabla f_{\alpha_{n}}\right)$ is nonexpansive for all $n \geq 0$ and $\lambda \in(0,2 / L)$. We observe that if $p \in \Omega$, then

$$
\left\|y_{n, 1}-p\right\| \leq\left\|u_{n}-p\right\| \leq\left\|x_{n}-p\right\| .
$$


For all, from $i=2$ to $i=N$, by induction, one proves that

$$
\begin{aligned}
\left\|y_{n, i}-p\right\| & \leq \beta_{n, i}\left\|u_{n}-p\right\|+\left(1-\beta_{n, i}\right)\left\|y_{n, i-1}-p\right\| \\
& \leq\left\|u_{n}-p\right\| \leq\left\|x_{n}-p\right\| .
\end{aligned}
$$

Thus, we obtain that for every $i=1, \ldots, N$,

$$
\left\|y_{n, i}-p\right\| \leq\left\|u_{n}-p\right\| \leq\left\|x_{n}-p\right\| \text {. }
$$

For simplicity, put $\tilde{y}_{n, N}=P_{C}\left(y_{n, N}-\lambda \nabla f_{\alpha_{n}}\left(y_{n, N}\right)\right)$ and $\tilde{y}_{n}=$ $P_{C}\left(y_{n}-\lambda \nabla f_{\alpha_{n}}\left(y_{n}\right)\right)$ for every $n \geq 0$. Then, $y_{n}=\alpha_{n} Q y_{n, N}+$ $\left(1-\alpha_{n}\right) \tilde{y}_{n, N}$ and $x_{n+1}=\sigma_{n} y_{n}+\gamma_{n} \widetilde{y}_{n}+\delta_{n} T \widetilde{y}_{n}$ for every $n \geq 0$. Taking into consideration that $T p=p$ and $P_{C}(I-\lambda \nabla f) p=p$ for $\lambda \in(0,2 / L)$, we have

$$
\begin{aligned}
& \left\|\tilde{y}_{n, N}-p\right\| \\
& =\left\|P_{C}\left(I-\lambda \nabla f_{\alpha_{n}}\right) y_{n, N}-P_{C}(I-\lambda \nabla f) p\right\| \\
& \leq\left\|P_{C}\left(I-\lambda \nabla f_{\alpha_{n}}\right) y_{n, N}-P_{C}\left(I-\lambda \nabla f_{\alpha_{n}}\right) p\right\| \\
& \quad+\left\|P_{C}\left(I-\lambda \nabla f_{\alpha_{n}}\right) p-P_{C}(I-\lambda \nabla f) p\right\| \\
& \leq\left\|y_{n, N}-p\right\|+\left\|P_{C}\left(I-\lambda \nabla f_{\alpha_{n}}\right) p-P_{C}(I-\lambda \nabla f) p\right\| \\
& \leq\left\|y_{n, N}-p\right\|+\lambda \alpha_{n}\|p\| .
\end{aligned}
$$

Similarly, we get $\left\|\tilde{y}_{n}-p\right\| \leq\left\|y_{n}-p\right\|+\lambda \alpha_{n}\|p\|$. Thus, from (34) we have

$$
\begin{aligned}
& \left\|y_{n}-p\right\|=\left\|\beta_{n}\left(Q y_{n, N}-p\right)+\left(1-\beta_{n}\right)\left(\tilde{y}_{n, N}-p\right)\right\| \\
& \leq \beta_{n}\left\|Q y_{n, N}-p\right\|+\left(1-\beta_{n}\right)\left\|\tilde{y}_{n, N}-p\right\| \\
& \leq \beta_{n}\left\|Q y_{n, N}-Q p\right\|+\beta_{n}\|Q p-p\| \\
& +\left(1-\beta_{n}\right)\left(\left\|y_{n, N}-p\right\|+\lambda \alpha_{n}\|p\|\right) \\
& \leq \beta_{n} \rho\left\|y_{n, N}-p\right\|+\beta_{n}\|Q p-p\| \\
& +\left(1-\beta_{n}\right)\left(\left\|y_{n, N}-p\right\|+\lambda \alpha_{n}\|p\|\right) \\
& =\left(1-\beta_{n}(1-\rho)\right)\left\|y_{n, N}-p\right\| \\
& +\beta_{n}\|Q p-p\|+\left(1-\beta_{n}\right) \lambda \alpha_{n}\|p\| \\
& =\left(1-\beta_{n}(1-\rho)\right)\left\|y_{n, N}-p\right\| \\
& +\beta_{n}(1-\rho) \frac{\|Q p-p\|}{1-\rho}+\left(1-\beta_{n}\right) \lambda \alpha_{n}\|p\| \\
& \leq \max \left\{\left\|y_{n, N}-p\right\|, \frac{\|Q p-p\|}{1-\rho}\right\}+\lambda \alpha_{n}\|p\| \\
& \leq \max \left\{\left\|u_{n}-p\right\|, \frac{\|Q p-p\|}{1-\rho}\right\}+\lambda \alpha_{n}\|p\| \\
& \leq \max \left\{\left\|x_{n}-p\right\|, \frac{\|Q p-p\|}{1-\rho}\right\}+\lambda \alpha_{n}\|p\| .
\end{aligned}
$$

Since $\left(\gamma_{n}+\delta_{n}\right) \zeta \leq \gamma_{n}$ for all $n \geq 0$, utilizing Lemma 10, we derive from (35)

$$
\begin{aligned}
& \left\|x_{n+1}-p\right\| \\
& \quad=\left\|\sigma\left(y_{n}-p\right)+\gamma_{n}\left(\tilde{y}_{n}-p\right)+\delta_{n}\left(T \tilde{y}_{n}-p\right)\right\| \\
& \quad \leq \sigma_{n}\left\|y_{n}-p\right\|+\left\|\gamma_{n}\left(\tilde{y}_{n}-p\right)+\delta_{n}\left(T \tilde{y}_{n}-p\right)\right\| \\
& \quad \leq \sigma_{n}\left\|y_{n}-p\right\|+\left(\gamma_{n}+\delta_{n}\right)\left\|\tilde{y}_{n}-p\right\| \\
& \quad \leq \sigma_{n}\left\|y_{n}-p\right\|+\left(\gamma_{n}+\delta_{n}\right)\left(\left\|y_{n}-p\right\|+\lambda \alpha_{n}\|p\|\right) \\
& \quad \leq\left\|y_{n}-p\right\|+\lambda \alpha_{n}\|p\| \\
& \quad \leq \max \left\{\left\|x_{n}-p\right\|, \frac{\|Q p-p\|}{1-\rho}\right\}+\lambda \alpha_{n}\|p\|+\lambda \alpha_{n}\|p\| \\
& \quad=\max \left\{\left\|x_{n}-p\right\|, \frac{\|Q p-p\|}{1-\rho}\right\}+2 \lambda \alpha_{n}\|p\| .
\end{aligned}
$$

By induction, we get

$$
\begin{aligned}
\left\|x_{n+1}-p\right\| \leq & \max \left\{\left\|x_{0}-p\right\|, \frac{\|Q p-p\|}{1-\rho}\right\} \\
& +2 \lambda\|p\| \cdot \sum_{i=0}^{n} \alpha_{i}, \quad \forall n \geq 0 .
\end{aligned}
$$

This implies that $\left\{x_{n}\right\}$ is bounded and so are $\left\{y_{n}\right\},\left\{u_{n}\right\}$, and $\left\{y_{n, i}\right\}$ for each $i=1, \ldots, N$. It is clear that both $\left\{\widetilde{y}_{n, N}\right\}$ and $\left\{\widetilde{y}_{n}\right\}$ are also bounded. Since $\left\|T \tilde{y}_{n}-p\right\| \leq((1+\zeta) /(1-\zeta))\left\|\tilde{y}_{n}-p\right\|$, $\left\{T \widetilde{y}_{n}\right\}$ is also bounded.

Lemma 16. Let one suppose that $\Omega \neq \emptyset$. Moreover, let one suppose that the following hold:

(H1) $\lim _{n \rightarrow \infty} \beta_{n}=0$ and $\sum_{n=0}^{\infty} \beta_{n}=\infty$;

(H2) $\sum_{n=1}^{\infty}\left|\beta_{n}-\beta_{n-1}\right|<\infty$ or $\lim _{n \rightarrow \infty}\left(\left|\beta_{n}-\beta_{n-1}\right| / \beta_{n}\right)=0$;

(H3) $\sum_{n=1}^{\infty}\left|\beta_{n, i}-\beta_{n-1, i}\right|<\infty$ or $\lim _{n \rightarrow \infty}\left(\left|\beta_{n, i}-\beta_{n-1, i}\right| / \beta_{n}\right)=$ 0 for each $i=1, \ldots, N$;

(H4) $\sum_{n=1}^{\infty}\left|r_{n}-r_{n-1}\right|<\infty$ or $\lim _{n \rightarrow \infty}\left(\left|r_{n}-r_{n-1}\right| / \beta_{n}\right)=0$;

(H5) $\sum_{n=1}^{\infty}\left|\sigma_{n}-\sigma_{n-1}\right|<\infty$ or $\lim _{n \rightarrow \infty}\left(\left|\sigma_{n}-\sigma_{n-1}\right| / \beta_{n}\right)=0$;

(H6) $\sum_{n=1}^{\infty}\left|\gamma_{n} /\left(1-\sigma_{n}\right)-\gamma_{n-1} /\left(1-\sigma_{n-1}\right)\right|<\infty$ or $\lim _{n \rightarrow \infty}\left(1 / \beta_{n}\right)\left|\gamma_{n} /\left(1-\sigma_{n}\right)-\gamma_{n-1} /\left(1-\sigma_{n-1}\right)\right|=0$.

Then, $\lim _{n \rightarrow \infty}\left\|x_{n+1}-x_{n}\right\|=0$, that is, $\left\{x_{n}\right\}$ is asymptotically regular.

Proof. Taking into account $0<\liminf _{n \rightarrow \infty} \sigma_{n} \leq$ lim $\sup _{n \rightarrow \infty} \sigma_{n}<1$, we may assume, without loss of generality, that $\left\{\sigma_{n}\right\} \subset[c, d]$ for some $c, d \in(0,1)$. First, we write 
$x_{n}=\sigma_{n-1} y_{n-1}+\left(1-\sigma_{n-1}\right) v_{n-1}$, for all $n \geq 1$, where $v_{n-1}=$ $\left(x_{n}-\sigma_{n-1} y_{n-1}\right) /\left(1-\sigma_{n-1}\right)$. It follows that for all $n \geq 1$

$$
\begin{aligned}
v_{n}-v_{n-1}= & \frac{x_{n+1}-\sigma_{n} y_{n}}{1-\sigma_{n}}-\frac{x_{n}-\sigma_{n-1} y_{n-1}}{1-\sigma_{n-1}} \\
= & \frac{\gamma_{n} \widetilde{y}_{n}+\delta_{n} T \widetilde{y}_{n}}{1-\sigma_{n}}-\frac{\gamma_{n-1} \tilde{y}_{n-1}+\delta_{n-1} T \tilde{y}_{n-1}}{1-\sigma_{n-1}} \\
= & \frac{\gamma_{n}\left(\tilde{y}_{n}-\tilde{y}_{n-1}\right)+\delta_{n}\left(T \widetilde{y}_{n}-T \tilde{y}_{n-1}\right)}{1-\sigma_{n}} \\
& +\left(\frac{\gamma_{n}}{1-\sigma_{n}}-\frac{\gamma_{n-1}}{1-\sigma_{n-1}}\right) \tilde{y}_{n-1} \\
& +\left(\frac{\delta_{n}}{1-\sigma_{n}}-\frac{\delta_{n-1}}{1-\sigma_{n-1}}\right) T \tilde{y}_{n-1} .
\end{aligned}
$$

Since $\left(\gamma_{n}+\delta_{n}\right) \zeta \leq \gamma_{n}$ for all $n \geq 0$, utilizing Lemma 10, we have

$$
\begin{gathered}
\left\|\gamma_{n}\left(\tilde{y}_{n}-\tilde{y}_{n-1}\right)+\delta_{n}\left(T \tilde{y}_{n}-T \tilde{y}_{n-1}\right)\right\| \\
\leq\left(\gamma_{n}+\delta_{n}\right)\left\|\tilde{y}_{n}-\tilde{y}_{n-1}\right\| .
\end{gathered}
$$

Next, we estimate $\left\|y_{n}-y_{n-1}\right\|$. Observe that for every $n \geq 1$

$$
\begin{aligned}
&\left\|\tilde{y}_{n, N}-\tilde{y}_{n-1, N}\right\| \\
& \leq\left\|P_{C}\left(I-\lambda \nabla f_{\alpha_{n}}\right) y_{n, N}-P_{C}\left(I-\lambda \nabla f_{\alpha_{n}}\right) y_{n-1, N}\right\| \\
&+\left\|P_{C}\left(I-\lambda \nabla f_{\alpha_{n}}\right) y_{n-1, N}-P_{C}\left(I-\lambda \nabla f_{\alpha_{n-1}}\right) y_{n-1, N}\right\| \\
& \leq\left\|y_{n, N}-y_{n-1, N}\right\| \\
&+\left\|P_{C}\left(I-\lambda \nabla f_{\alpha_{n}}\right) y_{n-1, N}-P_{C}\left(I-\lambda \nabla f_{\alpha_{n-1}}\right) y_{n-1, N}\right\| \\
& \leq\left\|y_{n, N}-y_{n-1, N}\right\| \\
&+\left\|\left(I-\lambda \nabla f_{\alpha_{n}}\right) y_{n-1, N}-\left(I-\lambda \nabla f_{\alpha_{n-1}}\right) y_{n-1, N}\right\| \\
&=\left\|y_{n, N}-y_{n-1, N}\right\|+\left\|\lambda \nabla f_{\alpha_{n}}\left(y_{n-1, N}\right)-\lambda \nabla f_{\alpha_{n-1}}\left(y_{n-1, N}\right)\right\| \\
&=\left\|y_{n, N}-y_{n-1, N}\right\|+\lambda\left|\alpha_{n}-\alpha_{n-1}\right|\left\|y_{n-1, N}\right\|,
\end{aligned}
$$

and similarly,

$$
\left\|\tilde{y}_{n}-\tilde{y}_{n-1}\right\| \leq\left\|y_{n}-y_{n-1}\right\|+\lambda\left|\alpha_{n}-\alpha_{n-1}\right|\left\|y_{n-1}\right\| .
$$

Also, from (30), we have

$$
\begin{gathered}
y_{n}=\beta_{n} Q y_{n, N}+\left(1-\beta_{n}\right) \tilde{y}_{n, N}, \\
y_{n-1}=\beta_{n-1} Q y_{n-1, N}+\left(1-\beta_{n-1}\right) \tilde{y}_{n-1, N},
\end{gathered}
$$

$\forall n \geq 1$.

Simple calculations show that

$$
\begin{aligned}
y_{n}-y_{n-1}= & \left(1-\beta_{n}\right)\left(\tilde{y}_{n, N}-\tilde{y}_{n-1, N}\right) \\
& +\left(\beta_{n}-\beta_{n-1}\right)\left(\mathrm{Q} y_{n-1, N}-\tilde{y}_{n-1, N}\right) \\
& +\beta_{n}\left(Q y_{n, N}-Q y_{n-1, N}\right) .
\end{aligned}
$$

Then, passing to the norm we get from (40) that

$$
\begin{aligned}
\| y_{n}- & y_{n-1} \| \\
\leq & \left(1-\beta_{n}\right)\left\|\tilde{y}_{n, N}-\tilde{y}_{n-1, N}\right\| \\
& +\left|\beta_{n}-\beta_{n-1}\right|\left\|Q y_{n-1, N}-\tilde{y}_{n-1, N}\right\|+\beta_{n}\left\|Q y_{n, N}-Q y_{n-1, N}\right\| \\
\leq & \left(1-\beta_{n}\right)\left(\left\|y_{n, N}-y_{n-1, N}\right\|+\lambda\left|\alpha_{n}-\alpha_{n-1}\right|\left\|y_{n-1, N}\right\|\right) \\
& +\left|\beta_{n}-\beta_{n-1}\right|\left\|Q y_{n-1, N}-\tilde{y}_{n-1, N}\right\| \\
& +\beta_{n} \rho\left\|y_{n, N}-y_{n-1, N}\right\| \\
\leq & \left(1-(1-\rho) \beta_{n}\right)\left\|y_{n, N}-y_{n-1, N}\right\| \\
& +\lambda\left|\alpha_{n}-\alpha_{n-1}\right|\left\|y_{n-1, N}\right\| \\
& +\left|\beta_{n}-\beta_{n-1}\right|\left\|Q y_{n-1, N}-\tilde{y}_{n-1, N}\right\| \\
\leq & \left(1-(1-\rho) \beta_{n}\right)\left\|y_{n, N}-y_{n-1, N}\right\| \\
& +M_{1}\left(\left|\alpha_{n}-\alpha_{n-1}\right|+\left|\beta_{n}-\beta_{n-1}\right|\right)
\end{aligned}
$$

where $\lambda\left\|y_{n, N}\right\|+\left\|Q y_{n, N}-\tilde{y}_{n, N}\right\| \leq M_{1}$, for all $n \geq 0$ for some $M_{1} \geq 0$. Furthermore, by the definition of $y_{n, i}$ one obtains that, for all $i=N, \ldots, 2$

$$
\begin{aligned}
\left\|y_{n, i}-y_{n-1, i}\right\| \leq & \beta_{n, i}\left\|u_{n}-u_{n-1}\right\| \\
& +\left\|S_{i} u_{n-1}-y_{n-1, i-1}\right\|\left|\beta_{n, i}-\beta_{n-1, i}\right| \\
& +\left(1-\beta_{n, i}\right)\left\|y_{n, i-1}-y_{n-1, i-1}\right\| .
\end{aligned}
$$

In the case of $i=1$, we have

$$
\begin{aligned}
&\left\|y_{n, 1}-y_{n-1,1}\right\| \\
& \leq \beta_{n, 1}\left\|u_{n}-u_{n-1}\right\| \\
&+\left\|S_{1} u_{n-1}-u_{n-1}\right\|\left|\beta_{n, 1}-\beta_{n-1,1}\right| \\
&+\left(1-\beta_{n, 1}\right)\left\|u_{n}-u_{n-1}\right\| \\
&=\left\|u_{n}-u_{n-1}\right\|+\left\|S_{1} u_{n-1}-u_{n-1}\right\|\left|\beta_{n, 1}-\beta_{n-1,1}\right| .
\end{aligned}
$$

Substituting (46) in all (45) type one obtains for $i=2, \ldots, N$

$$
\left\|y_{n, i}-y_{n-1, i}\right\| \leq\left\|u_{n}-u_{n-1}\right\|
$$

$$
\begin{aligned}
& +\sum_{k=2}^{i}\left\|S_{k} u_{n-1}-y_{n-1, k-1}\right\|\left|\beta_{n, k}-\beta_{n-1, k}\right| \\
& +\left\|S_{1} u_{n-1}-u_{n-1}\right\|\left|\beta_{n, 1}-\beta_{n-1,1}\right| .
\end{aligned}
$$


This together with (44) implies that

$$
\begin{aligned}
& \left\|y_{n}-y_{n-1}\right\| \\
& \leq\left(1-(1-\rho) \beta_{n}\right) \\
& \times\left[\left\|u_{n}-u_{n-1}\right\|\right. \\
& +\sum_{k=2}^{N}\left\|S_{k} u_{n-1}-y_{n-1, k-1}\right\|\left|\beta_{n, k}-\beta_{n-1, k}\right| \\
& \left.+\left\|S_{1} u_{n-1}-u_{n-1}\right\|\left|\beta_{n, 1}-\beta_{n-1,1}\right|\right] \\
& +M_{1}\left(\left|\alpha_{n}-\alpha_{n-1}\right|+\left|\beta_{n}-\beta_{n-1}\right|\right) \\
& \leq\left(1-(1-\rho) \beta_{n}\right)\left\|u_{n}-u_{n-1}\right\| \\
& +\sum_{k=2}^{N}\left\|S_{k} u_{n-1}-y_{n-1, k-1}\right\|\left|\beta_{n, k}-\beta_{n-1, k}\right| \\
& +\left\|S_{1} u_{n-1}-u_{n-1}\right\|\left|\beta_{n, 1}-\beta_{n-1,1}\right| \\
& +M_{1}\left(\left|\alpha_{n}-\alpha_{n-1}\right|+\left|\beta_{n}-\beta_{n-1}\right|\right) \text {. }
\end{aligned}
$$

By Lemma 13, we know that

$$
\left\|u_{n}-u_{n-1}\right\| \leq\left\|x_{n}-x_{n-1}\right\|+\kappa\left|1-\frac{r_{n-1}}{r_{n}}\right|
$$

where $\kappa=\sup _{n \geq 0}\left\|u_{n}-x_{n}\right\|$. So, substituting (49) in (48) we obtain

$$
\begin{aligned}
\| y_{n}- & y_{n-1} \| \\
\leq & \left(1-(1-\rho) \beta_{n}\right)\left(\left\|x_{n}-x_{n-1}\right\|+\kappa\left|1-\frac{r_{n-1}}{r_{n}}\right|\right) \\
& +\sum_{k=2}^{N}\left\|S_{k} u_{n-1}-y_{n-1, k-1}\right\|\left|\beta_{n, k}-\beta_{n-1, k}\right| \\
& +\left\|S_{1} u_{n-1}-u_{n-1}\right\|\left|\beta_{n, 1}-\beta_{n-1,1}\right| \\
& +M_{1}\left(\left|\alpha_{n}-\alpha_{n-1}\right|+\left|\beta_{n}-\beta_{n-1}\right|\right) \\
\leq & \left(1-(1-\rho) \beta_{n}\right)\left\|x_{n}-x_{n-1}\right\|+\kappa \frac{\left|r_{n}-r_{n-1}\right|}{r_{n}} \\
& +\sum_{k=2}^{N}\left\|S_{k} u_{n-1}-y_{n-1, k-1}\right\|\left|\beta_{n, k}-\beta_{n-1, k}\right| \\
& +\left\|S_{1} u_{n-1}-u_{n-1}\right\|\left|\beta_{n, 1}-\beta_{n-1,1}\right| \\
& +M_{1}\left(\left|\alpha_{n}-\alpha_{n-1}\right|+\left|\beta_{n}-\beta_{n-1}\right|\right) \\
\leq & \left(1-(1-\rho) \beta_{n}\right)\left\|x_{n}-x_{n-1}\right\|
\end{aligned}
$$

$$
\begin{gathered}
+M_{2}\left[\frac{\left|r_{n}-r_{n-1}\right|}{r_{n}}+\sum_{k=2}^{N}\left|\beta_{n, k}-\beta_{n-1, k}\right|+\left|\beta_{n, 1}-\beta_{n-1,1}\right|\right. \\
\left.\quad+\left|\alpha_{n}-\alpha_{n-1}\right|+\left|\beta_{n}-\beta_{n-1}\right|\right] \\
\leq\left(1-(1-\rho) \beta_{n}\right) \| x_{n}-x_{n-1}|| \\
+M_{2}\left[\frac{\left|r_{n}-r_{n-1}\right|}{b}+\sum_{k=1}^{N}\left|\beta_{n, k}-\beta_{n-1, k}\right|\right. \\
\left.+\left|\alpha_{n}-\alpha_{n-1}\right|+\left|\beta_{n}-\beta_{n-1}\right|\right],
\end{gathered}
$$

where $b>0$ is a minorant for $\left\{r_{n}\right\}$ and $\kappa+M_{1}+\sum_{k=2}^{N} \| S_{k} u_{n}-$ $y_{n, k-1}\|+\| S_{1} u_{n}-u_{n} \| \leq M_{2}$, for all $n \geq 0$ for some $M_{2} \geq 0$. This together with (38)-(39), implies that

$$
\begin{aligned}
& \left\|v_{n}-v_{n-1}\right\| \leq \frac{\left\|\gamma_{n}\left(\tilde{y}_{n}-\tilde{y}_{n-1}\right)+\delta_{n}\left(T \tilde{y}_{n}-T \tilde{y}_{n-1}\right)\right\|}{1-\sigma_{n}} \\
& +\left|\frac{\gamma_{n}}{1-\sigma_{n}}-\frac{\gamma_{n-1}}{1-\sigma_{n-1}}\right|\left\|\tilde{y}_{n-1}\right\| \\
& +\left|\frac{\delta_{n}}{1-\sigma_{n}}-\frac{\delta_{n-1}}{1-\sigma_{n-1}}\right|\left\|T \widetilde{y}_{n-1}\right\| \\
& \leq \frac{\left(\gamma_{n}+\delta_{n}\right)\left\|\tilde{y}_{n}-\tilde{y}_{n-1}\right\|}{1-\sigma_{n}} \\
& +\left|\frac{\gamma_{n}}{1-\sigma_{n}}-\frac{\gamma_{n-1}}{1-\sigma_{n-1}}\right|\left\|\tilde{y}_{n-1}\right\| \\
& +\left|\frac{\gamma_{n}}{1-\sigma_{n}}-\frac{\gamma_{n-1}}{1-\sigma_{n-1}}\right|\left\|T \tilde{y}_{n-1}\right\| \\
& =\left\|\tilde{y}_{n}-\tilde{y}_{n-1}\right\| \\
& +\left|\frac{\gamma_{n}}{1-\sigma_{n}}-\frac{\gamma_{n-1}}{1-\sigma_{n-1}}\right|\left(\left\|\tilde{y}_{n-1}\right\|+\left\|T \tilde{y}_{n-1}\right\|\right) \\
& \leq\left\|y_{n}-y_{n-1}\right\|+\lambda\left|\alpha_{n}-\alpha_{n-1}\right|\left\|y_{n-1}\right\| \\
& +\left|\frac{\gamma_{n}}{1-\sigma_{n}}-\frac{\gamma_{n-1}}{1-\sigma_{n-1}}\right|\left(\left\|\tilde{y}_{n-1}\right\|+\left\|T \tilde{y}_{n-1}\right\|\right) \\
& \leq\left(1-(1-\rho) \beta_{n}\right)\left\|x_{n}-x_{n-1}\right\| \\
& +M_{2}\left[\frac{\left|r_{n}-r_{n-1}\right|}{b}+\sum_{k=1}^{N}\left|\beta_{n, k}-\beta_{n-1, k}\right|\right. \\
& \left.+\left|\alpha_{n}-\alpha_{n-1}\right|+\left|\beta_{n}-\beta_{n-1}\right|\right] \\
& +\lambda\left|\alpha_{n}-\alpha_{n-1}\right|\left\|y_{n-1}\right\| \\
& +\left|\frac{\gamma_{n}}{1-\sigma_{n}}-\frac{\gamma_{n-1}}{1-\sigma_{n-1}}\right|\left(\left\|\tilde{y}_{n-1}\right\|+\left\|T \tilde{y}_{n-1}\right\|\right) \\
& \leq\left(1-(1-\rho) \beta_{n}\right)\left\|x_{n}-x_{n-1}\right\|
\end{aligned}
$$




$$
\begin{aligned}
+ & M_{3}\left[\frac{\left|r_{n}-r_{n-1}\right|}{b}+\sum_{k=1}^{N}\left|\beta_{n, k}-\beta_{n-1, k}\right|\right. \\
& \left.+\left|\alpha_{n}-\alpha_{n-1}\right|+\left|\beta_{n}-\beta_{n-1}\right|\right] \\
+ & M_{3}\left|\alpha_{n}-\alpha_{n-1}\right|+M_{3}\left|\frac{\gamma_{n}}{1-\sigma_{n}}-\frac{\gamma_{n-1}}{1-\sigma_{n-1}}\right| \\
=\left(1-(1-\rho) \beta_{n}\right)|| x_{n}-x_{n-1}|| & {\left[\frac{\left|r_{n}-r_{n-1}\right|}{b}+\sum_{k=1}^{N}\left|\beta_{n, k}-\beta_{n-1, k}\right|\right.} \\
+M_{3}\left[\begin{array}{l}
b \\
+
\end{array}\right. & +2\left|\alpha_{n-1}\right|+\left|\beta_{n}-\beta_{n-1}\right| \\
& \left.+\left|\frac{\gamma_{n}}{1-\sigma_{n}}-\frac{\gamma_{n-1}}{1-\sigma_{n-1}}\right|\right]
\end{aligned}
$$

where $M_{2}+\lambda\left\|y_{n}\right\|+\left\|\tilde{y}_{n}\right\|+\left\|T \tilde{y}_{n}\right\| \leq M_{3}$, for all $n \geq 0$ for some $M_{3} \geq 0$.

Further, we observe that

$$
\begin{gathered}
x_{n+1}=\sigma_{n} y_{n}+\left(1-\sigma_{n}\right) v_{n}, \\
x_{n}=\sigma_{n-1} y_{n-1}+\left(1-\beta_{n-1}\right) v_{n-1},
\end{gathered}
$$

$\forall n \geq 1$.

Simple calculations show that

$$
\begin{aligned}
x_{n+1}-x_{n}= & \left(1-\sigma_{n}\right)\left(v_{n}-v_{n-1}\right) \\
& +\left(\sigma_{n}-\sigma_{n-1}\right)\left(y_{n-1}-v_{n-1}\right) \\
& +\sigma_{n}\left(y_{n}-y_{n-1}\right) .
\end{aligned}
$$

Then, passing to the norm, we get from (51)

$$
\begin{aligned}
& \left\|x_{n+1}-x_{n}\right\| \\
& \leq\left(1-\sigma_{n}\right)\left\|v_{n}-v_{n-1}\right\| \\
& +\left|\sigma_{n}-\sigma_{n-1}\right|\left\|y_{n-1}-v_{n-1}\right\|+\sigma_{n}\left\|y_{n}-y_{n-1}\right\| \\
& \leq\left(1-\sigma_{n}\right) \\
& \quad \times\left\{\left(1-(1-\rho) \beta_{n}\right) \| x_{n}-x_{n-1}||\right. \\
& \quad+\quad\left[\frac{\left|r_{n}-r_{n-1}\right|}{b}+\sum_{k=1}^{N}\left|\beta_{n, k}-\beta_{n-1, k}\right|\right. \\
& \left.\left.\quad+\left|\frac{\gamma_{n}}{1-\sigma_{n}}-\frac{\gamma_{n-1}}{1-\sigma_{n-1}}\right|\right]\right\}
\end{aligned}
$$

$$
\begin{aligned}
& +\left|\sigma_{n}-\sigma_{n-1}\right|\left\|y_{n-1}-v_{n-1}\right\| \\
& +\sigma_{n}\left\{\left(1-(1-\rho) \beta_{n}\right)\left\|x_{n}-x_{n-1}\right\|\right. \\
& +M_{2}\left[\frac{\left|r_{n}-r_{n-1}\right|}{b}+\sum_{k=1}^{N}\left|\beta_{n, k}-\beta_{n-1, k}\right|\right. \\
& \left.\left.+\left|\alpha_{n}-\alpha_{n-1}\right|+\left|\beta_{n}-\beta_{n-1}\right|\right]\right\} \\
& \leq\left(1-(1-\rho) \beta_{n}\right)\left\|x_{n}-x_{n-1}\right\| \\
& +M_{3}\left[\frac{\left|r_{n}-r_{n-1}\right|}{b}+\sum_{k=1}^{N}\left|\beta_{n, k}-\beta_{n-1, k}\right|\right. \\
& +2\left|\alpha_{n}-\alpha_{n-1}\right|+\left|\beta_{n}-\beta_{n-1}\right| \\
& \left.+\left|\frac{\gamma_{n}}{1-\sigma_{n}}-\frac{\gamma_{n-1}}{1-\sigma_{n-1}}\right|\right] \\
& +\left|\sigma_{n}-\sigma_{n-1}\right|\left\|y_{n-1}-v_{n-1}\right\| \\
& \leq\left(1-(1-\rho) \beta_{n}\right)\left\|x_{n}-x_{n-1}\right\| \\
& +M\left[\frac{\left|r_{n}-r_{n-1}\right|}{b}+\sum_{k=1}^{N}\left|\beta_{n, k}-\beta_{n-1, k}\right|\right. \\
& +\left|\beta_{n}-\beta_{n-1}\right|+\left|\sigma_{n}-\sigma_{n-1}\right| \\
& \left.+\left|\frac{\gamma_{n}}{1-\sigma_{n}}-\frac{\gamma_{n-1}}{1-\sigma_{n-1}}\right|\right]+2 M\left|\alpha_{n}-\alpha_{n-1}\right|,
\end{aligned}
$$

where $M_{3}+\left\|y_{n}-v_{n}\right\| \leq M$, for all $n \geq 0$ for some $M \geq 0$. By hypotheses (H1)-(H6) and Lemma 9, from $\sum_{n=0}^{\infty} \alpha_{n}<\infty$, we obtain the claim.

Lemma 17. Let one suppose that $\Omega \neq \emptyset$. Let one suppose that $\left\{x_{n}\right\}$ is asymptotically regular. Then, $\left\|x_{n}-y_{n}\right\| \rightarrow 0$ and $\| x_{n}-$ $u_{n}\|=\| x_{n}-T_{r_{n}} x_{n} \| \rightarrow 0$ as $n \rightarrow \infty$.

Proof. We recall that, by the firm nonexpansivity of $T_{r_{n}}$, a standard calculation (see [17]) shows that if $v \in \operatorname{EP}(F, h)$, then

$$
\left\|u_{n}-v\right\|^{2} \leq\left\|x_{n}-v\right\|^{2}-\left\|x_{n}-u_{n}\right\|^{2}
$$

Let $p \in \Omega$. Then by Lemma 11, we have from (33)-(34) the following

$$
\begin{aligned}
\left\|\tilde{y}_{n}-p\right\|^{2} & =\left\|\beta_{n}\left(Q y_{n, N}-p\right)+\left(1-\beta_{n}\right)\left(\tilde{y}_{n, N}-p\right)\right\|^{2} \\
& \leq \beta_{n}\left\|Q y_{n, N}-p\right\|^{2}+\left(1-\beta_{n}\right)\left\|\tilde{y}_{n, N}-p\right\|^{2} \\
& \leq \beta_{n}\left\|Q y_{n, N}-p\right\|^{2}+\left\|\tilde{y}_{n, N}-p\right\|^{2} \\
& \leq \beta_{n}\left\|Q y_{n, N}-p\right\|^{2}+\left[\left\|y_{n, N}-p\right\|+\lambda \alpha_{n}\|p\|\right]^{2}
\end{aligned}
$$




$$
\begin{aligned}
= & \beta_{n}\left\|Q y_{n, N}-p\right\|^{2}+\left\|y_{n, N}-p\right\|^{2} \\
& +\lambda \alpha_{n}\|p\|\left(2\left\|y_{n, N}-p\right\|+\lambda \alpha_{n}\|p\|\right) \\
\leq & \beta_{n}\left\|Q y_{n, N}-p\right\|^{2}+\left\|x_{n}-p\right\|^{2} \\
& +\lambda \alpha_{n}\|p\|\left(2\left\|x_{n}-p\right\|+\lambda \alpha_{n}\|p\|\right), \\
\left\|\tilde{y}_{n}-p\right\|^{2}= & \left\|P_{C}\left(I-\lambda \nabla f_{\alpha_{n}}\right) y_{n}-P_{C}(I-\lambda \nabla f) p\right\|^{2} \\
\leq & \left(\left\|y_{n}-p\right\|+\lambda \alpha_{n}\|p\|\right)^{2} \\
= & \left\|y_{n}-p\right\|^{2}+\lambda \alpha_{n}\|p\|\left(2\left\|y_{n}-p\right\|+\lambda \alpha_{n}\|p\|\right) .
\end{aligned}
$$

Since $\left(\gamma_{n}+\delta_{n}\right) \zeta \leq \gamma_{n}$ for all $n \geq 0$, utilizing Lemma 10, we have

$$
\begin{aligned}
\left\|x_{n+1}-p\right\|^{2} & \left\|\sigma_{n}\left(y_{n}-p\right)+\gamma_{n}\left(\tilde{y}_{n}-p\right)+\delta_{n}\left(T \tilde{y}_{n}-p\right)\right\|^{2} \\
= & \| \sigma_{n}\left(y_{n}-p\right)+\left(\gamma_{n}+\delta_{n}\right) \frac{1}{\gamma_{n}+\delta_{n}} \\
& \times\left[\gamma_{n}\left(\tilde{y}_{n}-p\right)+\delta_{n}\left(T \tilde{y}_{n}-p\right)\right] \|^{2} \\
= & \sigma_{n}\left\|y_{n}-p\right\|^{2}+\left(\gamma_{n}+\delta_{n}\right) \\
& \times\left\|\frac{1}{\gamma_{n}+\delta_{n}}\left[\gamma_{n}\left(\tilde{y}_{n}-p\right)+\delta_{n}\left(T \tilde{y}_{n}-p\right)\right]\right\|^{2} \\
& -\sigma_{n}\left(\gamma_{n}+\delta_{n}\right) \|\left(y_{n}-p\right)-\frac{1}{\gamma_{n}+\delta_{n}} \\
= & \sigma_{n}\left\|y_{n}-p\right\|^{2}+\left(\gamma_{n}+\delta_{n}\right) \\
& \times\left\|\frac{1}{\gamma_{n}+\delta_{n}}\left[\gamma_{n}\left(\tilde{y}_{n}-p\right)+\delta_{n}\left(T \tilde{y}_{n}-p\right)\right]\right\|^{2} \\
& -\sigma_{n}\left(\gamma_{n}+\delta_{n}\right)\left\|\frac{1}{\gamma_{n}+\delta_{n}}\left[\gamma_{n}\left(\tilde{y}_{n}-y_{n}\right)+\delta_{n}\left(T \tilde{y}_{n}-y_{n}\right)\right]\right\|^{2} \\
= & \sigma_{n}\left\|y_{n}-p\right\|^{2}+\left(\gamma_{n}+\delta_{n}\right) \\
& \times\left\|\frac{1}{\gamma_{n}+\delta_{n}}\left[\gamma_{n}\left(\tilde{y}_{n}-p\right)+\delta_{n}\left(T \tilde{y}_{n}-p\right)\right]\right\|_{n}^{2}+\left(\tilde{y}_{n}+\delta_{n}\right)\left\|\tilde{y}_{n}-p\right\|^{2} \\
&
\end{aligned}
$$$$
\lim _{n \rightarrow \infty}\left\|x_{n}-y_{n}\right\|=0
$$$$
=\sigma_{n}\left\|y_{n}-p\right\|^{2}+\left(1-\sigma_{n}\right)\left\|\tilde{y}_{n}-p\right\|^{2}
$$$$
-\frac{\sigma_{n}}{1-\sigma_{n}}\left\|x_{n+1}-y_{n}\right\|^{2}
$$$$
\leq \sigma_{n}\left\|y_{n}-p\right\|^{2}+\left(1-\sigma_{n}\right)
$$$$
\times\left[\left\|y_{n}-p\right\|^{2}+\lambda \alpha_{n}\|p\|\left(2\left\|y_{n}-p\right\|+\lambda \alpha_{n}\|p\|\right)\right]
$$$$
-\frac{\sigma_{n}}{1-\sigma_{n}}\left\|x_{n+1}-y_{n}\right\|^{2}
$$$$
\leq\left\|y_{n}-p\right\|^{2}+\lambda \alpha_{n}\|p\|\left(2\left\|y_{n}-p\right\|+\lambda \alpha_{n}\|p\|\right)
$$$$
-\frac{\sigma_{n}}{1-\sigma_{n}}\left\|x_{n+1}-y_{n}\right\|^{2}
$$$$
\leq \beta_{n}\left\|Q y_{n, N}-p\right\|^{2}+\left\|x_{n}-p\right\|^{2}
$$$$
+\lambda \alpha_{n}\|p\|\left(2\left\|x_{n}-p\right\|+\lambda \alpha_{n}\|p\|\right)
$$$$
+\lambda \alpha_{n}\|p\|\left(2\left\|y_{n}-p\right\|+\lambda \alpha_{n}\|p\|\right)
$$$$
-\frac{\sigma_{n}}{1-\sigma_{n}}\left\|x_{n+1}-y_{n}\right\|^{2}
$$$$
=\left\|x_{n}-p\right\|^{2}+\beta_{n}\left\|Q y_{n, N}-p\right\|^{2}
$$$$
+2 \lambda \alpha_{n}\|p\|\left(\left\|x_{n}-p\right\|+\left\|y_{n}-p\right\|+\lambda \alpha_{n}\|p\|\right)
$$$$
-\frac{\sigma_{n}}{1-\sigma_{n}}\left\|x_{n+1}-y_{n}\right\|^{2} \text {. }
$$

Taking into account $0<\liminf _{n \rightarrow \infty} \sigma_{n} \leq \lim \sup _{n \rightarrow \infty} \sigma_{n}<$ 1 , we may assume that $\left\{\sigma_{n}\right\} \subset[c, d]$ for some $c, d \in(0,1)$. So, we deduce that

$$
\begin{aligned}
& \frac{c}{1-c}\left\|x_{n+1}-y_{n}\right\|^{2} \\
& \leq \frac{\sigma_{n}}{1-\sigma_{n}}\left\|x_{n+1}-y_{n}\right\|^{2} \\
& \leq \\
& \quad\left\|x_{n}-p\right\|^{2}-\left\|x_{n+1}-p\right\|^{2}+\beta_{n}\left\|Q y_{n, N}-p\right\|^{2} \\
& \quad+2 \lambda \alpha_{n}\|p\|\left(\left\|x_{n}-p\right\|+\left\|y_{n}-p\right\|+\lambda \alpha_{n}\|p\|\right) \\
& \leq\left(\left\|x_{n}-p\right\|+\left\|x_{n+1}-p\right\|\right)\left\|x_{n}-x_{n+1}\right\|+\beta_{n}\left\|Q y_{n, N}-p\right\|^{2} \\
& \quad+2 \lambda \alpha_{n}\|p\|\left(\left\|x_{n}-p\right\|+\left\|y_{n}-p\right\|+\lambda \alpha_{n}\|p\|\right) .
\end{aligned}
$$

Since $\alpha_{n} \rightarrow 0, \beta_{n} \rightarrow 0$ and $\left\|x_{n}-x_{n+1}\right\| \rightarrow 0$ as $n \rightarrow \infty$, we conclude from the boundedness of $\left\{x_{n}\right\},\left\{y_{n}\right\}$, and $\left\{y_{n, N}\right\}$ that $\left\|x_{n+1}-y_{n}\right\| \rightarrow 0$ as $n \rightarrow \infty$. This together with $\left\|x_{n}-x_{n+1}\right\| \rightarrow$ 0 , implies that 
Furthermore, from (33), (55), and (56), we have

$$
\begin{aligned}
\left\|y_{n}-p\right\|^{2} \leq & \beta_{n}\left\|Q y_{n, N}-p\right\|^{2}+\left\|y_{n, N}-p\right\|^{2} \\
& +\lambda \alpha_{n}\|p\|\left(2\left\|y_{n, N}-p\right\|+\lambda \alpha_{n}\|p\|\right) \\
\leq & \beta_{n}\left\|Q y_{n, N}-p\right\|^{2}+\left\|u_{n}-p\right\|^{2} \\
& +\lambda \alpha_{n}\|p\|\left(2\left\|y_{n, N}-p\right\|+\lambda \alpha_{n}\|p\|\right) \\
\leq & \beta_{n}\left\|Q y_{n, N}-p\right\|^{2}+\left\|x_{n}-p\right\|^{2}-\left\|x_{n}-u_{n}\right\|^{2} \\
& +\lambda \alpha_{n}\|p\|\left(2\left\|x_{n}-p\right\|+\lambda \alpha_{n}\|p\|\right),
\end{aligned}
$$

which hence implies that

$$
\begin{aligned}
\left\|x_{n}-u_{n}\right\|^{2} \leq & \beta_{n}\left\|Q y_{n, N}-p\right\|^{2}+\left\|x_{n}-p\right\|^{2}-\left\|y_{n}-p\right\|^{2} \\
& +\lambda \alpha_{n}\|p\|\left(2\left\|x_{n}-p\right\|+\lambda \alpha_{n}\|p\|\right) \\
\leq & \beta_{n}\left\|Q y_{n, N}-p\right\|^{2} \\
& +\left(\left\|x_{n}-p\right\|+\left\|y_{n}-p\right\|\right)\left\|x_{n}-y_{n}\right\| \\
& +\lambda \alpha_{n}\|p\|\left(2\left\|x_{n}-p\right\|+\lambda \alpha_{n}\|p\|\right) .
\end{aligned}
$$

Since $\alpha_{n} \rightarrow 0, \beta_{n} \rightarrow 0$ and $\left\|x_{n}-y_{n}\right\| \rightarrow 0$ as $n \rightarrow \infty$, we deduce from the boundedness of $\left\{x_{n}\right\},\left\{y_{n}\right\}$, and $\left\{y_{n, N}\right\}$ that

$$
\lim _{n \rightarrow \infty}\left\|x_{n}-u_{n}\right\|=0 \text {. }
$$

Remark 18. By the last lemma we have $\omega_{w}\left(x_{n}\right)=\omega_{w}\left(u_{n}\right)$ and $\omega_{s}\left(x_{n}\right)=\omega_{s}\left(u_{n}\right)$; that is, the sets of strong/weak cluster points of $\left\{x_{n}\right\}$ and $\left\{u_{n}\right\}$ coincide.

Of course, if $\beta_{n, i} \rightarrow \beta_{i} \neq 0$, as $n \rightarrow \infty$, for all index $i$, the assumptions of Lemma 16 are enough to assure that

$$
\lim _{n \rightarrow \infty} \frac{\left\|x_{n+1}-x_{n}\right\|}{\beta_{n, i}}=0, \quad \forall i \in\{1, \ldots, N\} .
$$

In the next lemma, we examine the case in which at least one sequence $\left\{\beta_{n, k_{0}}\right\}$ is a null sequence.

Lemma 19. Let one suppose that $\Omega \neq \emptyset$. Let one suppose that (H1) holds. Moreover, for an index $k_{0} \in\{1, \ldots, N\}$, $\lim _{n \rightarrow \infty} \beta_{n, k_{0}}=0$, and the following hold:

(H7) for all $i$,

$$
\begin{aligned}
\lim _{n \rightarrow \infty} \frac{\left|\beta_{n, i}-\beta_{n-1, i}\right|}{\beta_{n} \beta_{n, k_{0}}} & =\lim _{n \rightarrow \infty} \frac{\left|\alpha_{n}-\alpha_{n-1}\right|}{\beta_{n} \beta_{n, k_{0}}}=\lim _{n \rightarrow \infty} \frac{\left|\beta_{n}-\beta_{n-1}\right|}{\beta_{n} \beta_{n, k_{0}}} \\
& =\lim _{n \rightarrow \infty} \frac{\left|\sigma_{n}-\sigma_{n-1}\right|}{\beta_{n} \beta_{n, k_{0}}}=\lim _{n \rightarrow \infty} \frac{\left|r_{n}-r_{n-1}\right|}{\beta_{n} \beta_{n, k_{0}}} \\
& =\lim _{n \rightarrow \infty} \frac{1}{\beta_{n} \beta_{n, k_{0}}}\left|\frac{\gamma_{n}}{1-\sigma_{n}}-\frac{\gamma_{n-1}}{1-\sigma_{n-1}}\right|=0 ;
\end{aligned}
$$

(H8) there exists a constant $\tau>0$ such that $\left(1 / \beta_{n}\right) \mid 1 / \beta_{n, k_{0}}-$ $1 / \beta_{n-1, k_{0}} \mid<\tau$ for all $n \geq 1$. Then,

$$
\lim _{n \rightarrow \infty} \frac{\left\|x_{n+1}-x_{n}\right\|}{\beta_{n, k_{0}}}=0
$$

Proof. We start by (54). Dividing both the terms by $\beta_{n, k_{0}}$ we have

$$
\frac{\left\|x_{n+1}-x_{n}\right\|}{\beta_{n, k_{0}}}
$$

$$
\begin{gathered}
\leq\left[1-(1-\rho) \beta_{n}\right] \frac{\left\|x_{n}-x_{n-1}\right\|}{\beta_{n, k_{0}}} \\
+M\left[\frac{\left|r_{n}-r_{n-1}\right|}{b \beta_{n, k_{0}}}+\frac{\sum_{k=1}^{N}\left|\beta_{n, k}-\beta_{n-1, k}\right|}{\beta_{n, k_{0}}}\right. \\
+\frac{2\left|\alpha_{n}-\alpha_{n-1}\right|}{\beta_{n, k_{0}}}+\frac{\left|\beta_{n}-\beta_{n-1}\right|}{\beta_{n, k_{0}}}+\frac{\left|\sigma_{n}-\sigma_{n-1}\right|}{\beta_{n, k_{0}}} \\
\left.+\frac{\left|\gamma_{n} /\left(1-\sigma_{n}\right)-\gamma_{n-1} /\left(1-\sigma_{n-1}\right)\right|}{\beta_{n, k_{0}}}\right] .
\end{gathered}
$$

So, by (H8) we have

$$
\begin{aligned}
& \frac{\left\|x_{n+1}-x_{n}\right\|}{\beta_{n, k_{0}}} \\
& \leq\left[1-(1-\rho) \beta_{n}\right] \frac{\left\|x_{n}-x_{n-1}\right\|}{\beta_{n-1, k_{0}}} \\
& +\left[1-(1-\rho) \beta_{n}\right]\left\|x_{n}-x_{n-1}\right\|\left|\frac{1}{\beta_{n, k_{0}}}-\frac{1}{\beta_{n-1, k_{0}}}\right| \\
& +M\left[\frac{\left|r_{n}-r_{n-1}\right|}{b \beta_{n, k_{0}}}+\frac{\sum_{k=1}^{N}\left|\beta_{n, k}-\beta_{n-1, k}\right|}{\beta_{n, k_{0}}}\right. \\
& +\frac{\left|\gamma_{n} /\left(1-\sigma_{n}\right)-\gamma_{n-1} /\left(1-\sigma_{n-1}\right)\right|}{\beta_{n, k_{0}}} \\
& \left.+\frac{2\left|\alpha_{n}-\alpha_{n-1}\right|}{\beta_{n, k_{0}}}+\frac{\left|\beta_{n}-\beta_{n-1}\right|}{\beta_{n, k_{0}}}+\frac{\left|\sigma_{n}-\sigma_{n-1}\right|}{\beta_{n, k_{0}}}\right] \\
& \leq\left[1-(1-\rho) \beta_{n}\right] \frac{\left\|x_{n}-x_{n-1}\right\|}{\beta_{n-1, k_{0}}} \\
& +\left\|x_{n}-x_{n-1}\right\|\left|\frac{1}{\beta_{n, k_{0}}}-\frac{1}{\beta_{n-1, k_{0}}}\right| \\
& +M\left[\frac{\left|r_{n}-r_{n-1}\right|}{b \beta_{n, k_{0}}}+\frac{\sum_{k=1}^{N}\left|\beta_{n, k}-\beta_{n-1, k}\right|}{\beta_{n, k_{0}}}\right.
\end{aligned}
$$




$$
\begin{aligned}
& +\frac{\left|\gamma_{n} /\left(1-\sigma_{n}\right)-\gamma_{n-1} /\left(1-\sigma_{n-1}\right)\right|}{\beta_{n, k_{0}}} \\
& +\frac{2\left|\alpha_{n}-\alpha_{n-1}\right|}{\beta_{n, k_{0}}} \\
& \left.+\frac{\left|\beta_{n}-\beta_{n-1}\right|}{\beta_{n, k_{0}}}+\frac{\left|\sigma_{n}-\sigma_{n-1}\right|}{\beta_{n, k_{0}}}\right] \\
& \leq\left[1-(1-\rho) \beta_{n}\right] \frac{\left\|x_{n}-x_{n-1}\right\|}{\beta_{n-1, k_{0}}}+\beta_{n} \tau\left\|x_{n}-x_{n-1}\right\| \\
& +M\left[\frac{\left|r_{n}-r_{n-1}\right|}{b \beta_{n, k_{0}}}+\frac{\sum_{k=1}^{N}\left|\beta_{n, k}-\beta_{n-1, k}\right|}{\beta_{n, k_{0}}}\right. \\
& +\frac{\left|\gamma_{n} /\left(1-\sigma_{n}\right)-\gamma_{n-1} /\left(1-\sigma_{n-1}\right)\right|}{\beta_{n, k_{0}}} \\
& \left.+\frac{2\left|\alpha_{n}-\alpha_{n-1}\right|}{\beta_{n, k_{0}}}+\frac{\left|\beta_{n}-\beta_{n-1}\right|}{\beta_{n, k_{0}}}+\frac{\left|\sigma_{n}-\sigma_{n-1}\right|}{\beta_{n, k_{0}}}\right] \\
& =\left[1-(1-\rho) \beta_{n}\right] \frac{\left\|x_{n}-x_{n-1}\right\|}{\beta_{n-1, k_{0}}}+(1-\rho) \beta_{n} \cdot \frac{1}{1-\rho} \\
& \times\left\{\tau\left\|x_{n}-x_{n-1}\right\|\right. \\
& +M\left[\frac{\left|r_{n}-r_{n-1}\right|}{b \beta_{n} \beta_{n, k_{0}}}+\frac{\sum_{k=1}^{N}\left|\beta_{n, k}-\beta_{n-1, k}\right|}{\beta_{n} \beta_{n, k_{0}}}\right. \\
& +\frac{\left|\gamma_{n} /\left(1-\sigma_{n}\right)-\gamma_{n-1} /\left(1-\sigma_{n-1}\right)\right|}{\beta_{n} \beta_{n, k_{0}}} \\
& +\frac{2\left|\alpha_{n}-\alpha_{n-1}\right|}{\beta_{n} \beta_{n, k_{0}}}+\frac{\left|\beta_{n}-\beta_{n-1}\right|}{\beta_{n} \beta_{n, k_{0}}} \\
& \left.\left.+\frac{\left|\sigma_{n}-\sigma_{n-1}\right|}{\beta_{n} \beta_{n, k_{0}}}\right]\right\} \text {. }
\end{aligned}
$$

Therefore, utilizing Lemma 9, from (H1), (H7), and the asymptotical regularity of $\left\{x_{n}\right\}$ (due to Lemma 16), we deduce that

$$
\lim _{n \rightarrow \infty} \frac{\left\|x_{n+1}-x_{n}\right\|}{\beta_{n, k_{0}}}=0
$$

Lemma 20. Let one suppose that $\Omega \neq \emptyset$. Let one suppose that (H1)-(H6) hold. Then,

$$
\lim _{n \rightarrow \infty}\left\|\widetilde{y}_{n, N}-y_{n, N}\right\|=\lim _{n \rightarrow \infty}\left\|\widetilde{y}_{n}-y_{n}\right\|=0 .
$$

Proof. Let $p \in \Omega$. Then, by Lemma 11 we have

$$
\begin{aligned}
\| y_{n}- & p \|^{2} \\
= & \left\|\beta_{n}\left(Q y_{n, N}-p\right)+\left(1-\beta_{n}\right)\left(\tilde{y}_{n, N}-p\right)\right\|^{2} \\
\leq & \beta_{n}\left\|Q y_{n, N}-p\right\|^{2}+\left(1-\beta_{n}\right)\left\|\widetilde{y}_{n, N}-p\right\|^{2} \\
= & \beta_{n}\left\|Q y_{n, N}-p\right\|^{2}+\left(1-\beta_{n}\right) \\
& \times\left\|P_{C}\left(I-\lambda \nabla f_{\alpha_{n}}\right) y_{n, N}-P_{C}(I-\lambda \nabla f) p\right\|^{2} \\
\leq & \beta_{n}\left\|Q y_{n, N}-p\right\|^{2}+\left(1-\beta_{n}\right) \\
& \times\left\|(I-\lambda \nabla f) y_{n, N}-(I-\lambda \nabla f) p-\lambda \alpha_{n} y_{n, N}\right\|^{2} \\
\leq & \beta_{n}\left\|Q y_{n, N}-p\right\|^{2}+\left(1-\beta_{n}\right) \\
& \times\left[\left\|(I-\lambda \nabla f) y_{n, N}-(I-\lambda \nabla f) p\right\|^{2}\right. \\
& \left.-2 \lambda \alpha_{n}\left\langle y_{n, N},\left(I-\lambda \nabla f_{\alpha_{n}}\right) y_{n, N}-(I-\lambda \nabla f) p\right\rangle\right] \\
\leq & \beta_{n}\left\|Q y_{n, N}-p\right\|^{2}+\left(1-\beta_{n}\right) \\
& \times\left[\left\|y_{n, N}-p\right\|^{2}+2 \lambda\left(\lambda-\frac{2}{L}\right)\left\|\nabla f\left(y_{n, N}\right)-\nabla f(p)\right\|^{2}\right. \\
& \left.\quad+2 \lambda \alpha_{n}\left\|y_{n, N}\right\|\left\|\left(I-\lambda \nabla f_{\alpha_{n}}\right) y_{n, N}-(I-\lambda \nabla f) p\right\|\right] \\
\leq & \beta_{n}\left\|Q y_{n, N}-p\right\|^{2}+\left(1-\beta_{n}\right) \\
& \times\left[\left\|x_{n}-p\right\|^{2}+2 \lambda\left(\lambda-\frac{2}{L}\right)\left\|\nabla f\left(y_{n, N}\right)-\nabla f(p)\right\|^{2}\right. \\
& \left.+2 \lambda \alpha_{n}\left\|y_{n, N}\right\|\left\|\left(I-\lambda \nabla f_{\alpha_{n}}\right) y_{n, N}-(I-\lambda \nabla f) p\right\|\right] .
\end{aligned}
$$

So, we obtain

$$
\begin{aligned}
(1- & \left.\beta_{n}\right) 2 \lambda\left(\frac{2}{L}-\lambda\right)\left\|\nabla f\left(y_{n, N}\right)-\nabla f(p)\right\|^{2} \\
\leq & \beta_{n}\left\|Q y_{n, N}-p\right\|^{2}+\left(1-\beta_{n}\right)\left\|x_{n}-p\right\|^{2}-\left\|y_{n}-p\right\|^{2} \\
& +\left(1-\beta_{n}\right) 2 \lambda \alpha_{n}\left\|y_{n, N}\right\| \\
& \times\left\|\left(I-\lambda \nabla f_{\alpha_{n}}\right) y_{n, N}-(I-\lambda \nabla f) p\right\| \\
\leq & \beta_{n}\left\|Q y_{n, N}-p\right\|^{2}+\left(\left\|x_{n}-p\right\|+\left\|y_{n}-p\right\|\right) \\
& \times\left(\left\|x_{n}-p\right\|-\left\|y_{n}-p\right\|\right) \\
& +2 \lambda \alpha_{n}\left\|y_{n, N}\right\|\left\|\left(I-\lambda \nabla f_{\alpha_{n}}\right) y_{n, N}-(I-\lambda \nabla f) p\right\| \\
\leq & \beta_{n}\left\|Q y_{n, N}-p\right\|^{2}+\left(\left\|x_{n}-p\right\|+\left\|y_{n}-p\right\|\right)\left(\left\|x_{n}-y_{n}\right\|\right) \\
& +2 \lambda \alpha_{n}\left\|y_{n, N}\right\|\left\|\left(I-\lambda \nabla f_{\alpha_{n}}\right) y_{n, N}-(I-\lambda \nabla f) p\right\| .
\end{aligned}
$$


Since $\alpha_{n} \rightarrow 0, \beta_{n} \rightarrow 0,\left\|x_{n}-y_{n}\right\| \rightarrow 0$, and $0<\lambda<2 / L$, from the boundedness of $\left\{x_{n}\right\},\left\{y_{n}\right\}$, and $\left\{y_{n, N}\right\}$ it follows that $\lim _{n \rightarrow \infty}\left\|\nabla f\left(y_{n, N}\right)-\nabla f(p)\right\|=0$, and hence

$$
\lim _{n \rightarrow \infty}\left\|\nabla f_{\alpha_{n}}\left(y_{n, N}\right)-\nabla f(p)\right\|=0 .
$$

Moreover, from the firm nonexpansiveness of $P_{C}$ we obtain

$$
\begin{aligned}
& \left\|\tilde{y}_{n, N}-p\right\|^{2} \\
& =\left\|P_{C}\left(I-\lambda \nabla f_{\alpha_{n}}\right) y_{n, N}-P_{C}(I-\lambda \nabla f) p\right\|^{2} \\
& \leq\left\langle\left(I-\lambda \nabla f_{\alpha_{n}}\right) y_{n, N}-(I-\lambda \nabla f) p, \tilde{y}_{n, N}-p\right\rangle \\
& =\frac{1}{2}\left\{\left\|\left(I-\lambda \nabla f_{\alpha_{n}}\right) y_{n, N}-(I-\lambda \nabla f) p\right\|^{2}+\left\|\tilde{y}_{n, N}-p\right\|^{2}\right. \\
& \left.\quad-\left\|\left(I-\lambda \nabla f_{\alpha_{n}}\right) y_{n, N}-(I-\lambda \nabla f) p-\left(\tilde{y}_{n, N}-p\right)\right\|^{2}\right\} \\
& \leq \frac{1}{2}\left\{\left\|y_{n, N}-p\right\|^{2}+2 \lambda\left\|\nabla f_{\alpha_{n}}\left(y_{n, N}\right)-\nabla f(p)\right\|\right. \\
& \quad \times\left\|\left(I-\lambda \nabla f_{\alpha_{n}}\right) y_{n, N}-(I-\lambda \nabla f) p\right\| \\
& \quad+\left\|\widetilde{y}_{n, N}-p\right\|^{2}-\left\|y_{n, N}-\tilde{y}_{n, N}\right\|^{2} \\
& +2 \lambda\left\langle y_{n, N}-\widetilde{y}_{n, N}, \nabla f_{\alpha_{n}}\left(y_{n, N}\right)-\nabla f(p)\right\rangle \\
& \left.\quad-\lambda^{2}\left\|\nabla f_{\alpha_{n}}\left(y_{n, N}\right)-\nabla f(p)\right\|^{2}\right\},
\end{aligned}
$$

and so

$$
\begin{aligned}
\left\|\tilde{y}_{n, N}-p\right\|^{2} \leq & \left\|y_{n, N}-p\right\|^{2}-\left\|y_{n, N}-\tilde{y}_{n, N}\right\|^{2} \\
& +2 \lambda\left\|\nabla f_{\alpha_{n}}\left(y_{n, N}\right)-\nabla f(p)\right\| \\
& \times \\
& \left\|\left(I-\lambda \nabla f_{\alpha_{n}}\right) y_{n, N}-(I-\lambda \nabla f) p\right\| \\
& +2 \lambda\left\langle y_{n, N}-\tilde{y}_{n, N}, \nabla f_{\alpha_{n}}\left(y_{n, N}\right)-\nabla f(p)\right\rangle \\
& -\lambda^{2}\left\|\nabla f_{\alpha_{n}}\left(y_{n, N}\right)-\nabla f(p)\right\|^{2} .
\end{aligned}
$$

Thus, we have

$$
\begin{aligned}
\left\|y_{n}-p\right\|^{2} \leq & \beta_{n}\left\|Q y_{n, N}-p\right\|^{2}+\left(1-\beta_{n}\right)\left\|\tilde{y}_{n, N}-p\right\|^{2} \\
\leq & \beta_{n}\left\|Q y_{n, N}-p\right\|^{2}+\left\|y_{n, N}-p\right\|^{2} \\
& -\left(1-\beta_{n}\right)\left\|y_{n, N}-\tilde{y}_{n, N}\right\|^{2} \\
& +2 \lambda\left\|\nabla f_{\alpha_{n}}\left(y_{n, N}\right)-\nabla f(p)\right\| \\
& \times \\
& \left\|\left(I-\lambda \nabla f_{\alpha_{n}}\right) y_{n, N}-(I-\lambda \nabla f) p\right\| \\
& +2\left(1-\beta_{n}\right) \lambda\left\langle y_{n, N}-\tilde{y}_{n, N}, \nabla f_{\alpha_{n}}\left(y_{n, N}\right)-\nabla f(p)\right\rangle \\
& -\left(1-\beta_{n}\right) \lambda^{2}\left\|\nabla f_{\alpha_{n}}\left(y_{n, N}\right)-\nabla f(p)\right\|^{2},
\end{aligned}
$$

which implies that

$$
\begin{aligned}
&\left(1-\beta_{n}\right)\left\|y_{n, N}-\tilde{y}_{n, N}\right\|^{2} \\
& \leq \beta_{n}\left\|Q y_{n, N}-p\right\|^{2}+\left\|y_{n, N}-p\right\|^{2} \\
&-\left\|y_{n}-p\right\|^{2}+2 \lambda\left\|\nabla f_{\alpha_{n}}\left(y_{n, N}\right)-\nabla f(p)\right\| \\
& \times\left\|\left(I-\lambda \nabla f_{\alpha_{n}}\right) y_{n, N}-(I-\lambda \nabla f) p\right\| \\
&+2\left(1-\beta_{n}\right) \lambda\left\langle y_{n, N}-\widetilde{y}_{n, N}, \nabla f_{\alpha_{n}}\left(y_{n, N}\right)-\nabla f(p)\right\rangle \\
&-\left(1-\beta_{n}\right) \lambda^{2}\left\|\nabla f_{\alpha_{n}}\left(y_{n, N}\right)-\nabla f(p)\right\|^{2} \\
& \leq \beta_{n}\left\|Q y_{n, N}-p\right\|^{2}+\left\|x_{n}-p\right\|^{2} \\
&-\left\|y_{n}-p\right\|^{2}+2 \lambda\left\|\nabla f_{\alpha_{n}}\left(y_{n, N}\right)-\nabla f(p)\right\| \\
& \times\left\|\left(I-\lambda \nabla f_{\alpha_{n}}\right) y_{n, N}-(I-\lambda \nabla f) p\right\| \\
&+2\left(1-\beta_{n}\right) \lambda\left\langle y_{n, N}-\widetilde{y}_{n, N}, \nabla f_{\alpha_{n}}\left(y_{n, N}\right)-\nabla f(p)\right\rangle \\
&-\left(1-\beta_{n}\right) \lambda^{2}\left\|\nabla f_{\alpha_{n}}\left(y_{n, N}\right)-\nabla f(p)\right\|^{2} \\
& \leq \beta_{n}\left\|Q y_{n, N}-p\right\|^{2}+\left(\left\|x_{n}-p\right\|+\left\|y_{n}-p\right\|\right)\left\|x_{n}-y_{n}\right\| \\
&+2 \lambda\left\|\nabla f_{\alpha_{n}}\left(y_{n, N}\right)-\nabla f(p)\right\| \\
& \times\left\|\left(I-\lambda \nabla f_{\alpha_{n}}\right) y_{n, N}-(I-\lambda \nabla f) p\right\| \\
&+2 \lambda\left\|y_{n, N}-\tilde{y}_{n, N}\right\|\left\|\nabla f_{\alpha_{n}}\left(y_{n, N}\right)-\nabla f(p)\right\| .
\end{aligned}
$$

Since $\beta_{n} \rightarrow 0,\left\|x_{n}-y_{n}\right\| \rightarrow 0$, and $\left\|\nabla f_{\alpha_{n}}\left(y_{n, N}\right)-\nabla f(p)\right\| \rightarrow$ 0 , from the boundedness of $\left\{x_{n}\right\},\left\{y_{n}\right\},\left\{y_{n, N}\right\}$, and $\left\{\tilde{y}_{n, N}\right\}$, it follows that $\lim _{n \rightarrow \infty}\left\|y_{n, N}-\widetilde{y}_{n, N}\right\|=0$. Observe that

$$
\left\|y_{n}-\tilde{y}_{n, N}\right\|=\beta_{n}\left\|Q y_{n, N}-\tilde{y}_{n, N}\right\| \longrightarrow 0 \quad \text { as } n \longrightarrow \infty,
$$

and hence

$$
\begin{aligned}
& \left\|\tilde{y}_{n}-y_{n}\right\| \\
& \quad \leq\left\|\tilde{y}_{n}-\tilde{y}_{n, N}\right\|+\left\|\tilde{y}_{n, N}-y_{n}\right\| \\
& \quad=\left\|P_{C}\left(I-\lambda \nabla f_{\alpha_{n}}\right) y_{n}-P_{C}\left(I-\lambda \nabla f_{\alpha_{n}}\right) y_{n, N}\right\|+\left\|\tilde{y}_{n, N}-y_{n}\right\| \\
& \quad \leq\left\|y_{n}-y_{n, N}\right\|+\left\|\tilde{y}_{n, N}-y_{n}\right\| \\
& \quad \leq\left\|y_{n}-\tilde{y}_{n, N}\right\|+\left\|\tilde{y}_{n, N}-y_{n, N}\right\|+\left\|\tilde{y}_{n, N}-y_{n}\right\| \\
& \quad=2\left\|y_{n}-\tilde{y}_{n, N}\right\|+\left\|\tilde{y}_{n, N}-y_{n, N}\right\| \longrightarrow 0 \quad \text { as } n \longrightarrow \infty .
\end{aligned}
$$

Thus, $\lim _{n \rightarrow \infty}\left\|\tilde{y}_{n}-y_{n}\right\|=0$.

Lemma 21. Let one suppose that $\Omega \neq \emptyset$. Let one suppose that $0<\liminf _{n \rightarrow \infty} \beta_{n, i} \leq \lim \sup _{n \rightarrow \infty} \beta_{n, i}<1$ for each $i=$ $1, \ldots, N$. Moreover, suppose that (H1)-(H6) are satisfied. Then, $\lim _{n \rightarrow \infty}\left\|S_{i} u_{n}-u_{n}\right\|=0$ for each $i=1, \ldots, N$. 
Proof. First of all, observe that

$$
\begin{aligned}
x_{n+1}-y_{n} & =\gamma_{n}\left(\tilde{y}_{n}-y_{n}\right)+\delta_{n}\left(T \tilde{y}_{n}-y_{n}\right) \\
& =\gamma_{n}\left(\tilde{y}_{n}-y_{n}\right)+\delta_{n}\left(T \tilde{y}_{n}-T y_{n}\right)+\delta_{n}\left(T y_{n}-y_{n}\right) .
\end{aligned}
$$

By Lemmas 16 and 20, we know that $\left\|x_{n+1}-x_{n}\right\| \rightarrow 0$ and $\left\|\widetilde{y}_{n}-y_{n}\right\| \rightarrow 0$ as $n \rightarrow \infty$. Hence, utilizing Lemma 7(i), we have

$$
\begin{aligned}
& \left\|\delta_{n}\left(T y_{n}-y_{n}\right)\right\| \\
& \quad=\left\|x_{n+1}-y_{n}-\gamma_{n}\left(\tilde{y}_{n}-y_{n}\right)-\delta_{n}\left(T \tilde{y}_{n}-T y_{n}\right)\right\| \\
& \quad \leq\left\|x_{n+1}-y_{n}\right\|+\gamma_{n}\left\|\tilde{y}_{n}-y_{n}\right\|+\delta_{n}\left\|T \tilde{y}_{n}-T y_{n}\right\| \\
& \quad \leq\left\|x_{n+1}-y_{n}\right\|+\left\|\tilde{y}_{n}-y_{n}\right\|+\left\|T \tilde{y}_{n}-T y_{n}\right\| \\
& \quad \leq\left\|x_{n+1}-x_{n}\right\|+\left\|x_{n}-y_{n}\right\|+\left\|\tilde{y}_{n}-y_{n}\right\|+\frac{1+\zeta}{1-\zeta}\left\|\tilde{y}_{n}-y_{n}\right\| \\
& \quad=\left\|x_{n+1}-x_{n}\right\|+\left\|x_{n}-y_{n}\right\|+\frac{2}{1-\zeta}\left\|\tilde{y}_{n}-y_{n}\right\|,
\end{aligned}
$$

which together with $\left\|x_{n}-y_{n}\right\| \rightarrow 0$ implies that $\lim _{n \rightarrow \infty}\left\|\delta_{n}\left(T y_{n}-y_{n}\right)\right\|=0$. Taking into account $\liminf _{n \rightarrow \infty} \delta_{n}>0$, we have

$$
\lim _{n \rightarrow \infty}\left\|T y_{n}-y_{n}\right\|=0
$$

Let us show that for each $i \in\{1, \ldots, N\}$, one has $\| S_{i} u_{n}-$ $y_{n, i-1} \| \rightarrow 0$ as $n \rightarrow \infty$. Let $p \in \Omega$. When $i=N$, by Lemma 11, we have from (33)-(34) the following:

$$
\begin{aligned}
\left\|y_{n}-p\right\|^{2} \leq & \beta_{n}\left\|Q y_{n, N}-p\right\|^{2}+\left(1-\beta_{n}\right)\left\|\tilde{y}_{n, N}-p\right\|^{2} \\
\leq & \beta_{n}\left\|Q y_{n, N}-p\right\|^{2}+\left\|\tilde{y}_{n, N}-p\right\|^{2} \\
\leq & \beta_{n}\left\|Q y_{n, N}-p\right\|^{2}+\left(\left\|y_{n, N}-p\right\|+\lambda \alpha_{n}\|p\|\right)^{2} \\
= & \beta_{n}\left\|Q y_{n, N}-p\right\|^{2}+\left\|y_{n, N}-p\right\|^{2} \\
& +\lambda \alpha_{n}\|p\|\left(2\left\|y_{n, N}-p\right\|+\lambda \alpha_{n}\|p\|\right) \\
= & \beta_{n}\left\|Q y_{n, N}-p\right\|^{2}+\beta_{n, N}\left\|S_{N} u_{n}-p\right\|^{2} \\
& +\left(1-\beta_{n, N}\right)\left\|y_{n, N-1}-p\right\|^{2} \\
& -\beta_{n, N}\left(1-\beta_{n, N}\right)\left\|S_{N} u_{n}-y_{n, N-1}\right\|^{2} \\
& +\lambda \alpha_{n}\|p\|\left(2\left\|y_{n, N}-p\right\|+\lambda \alpha_{n}\|p\|\right) \\
\leq & \beta_{n}\left\|Q y_{n, N}-p\right\|^{2}+\left\|u_{n}-p\right\|^{2} \\
& -\beta_{n, N}\left(1-\beta_{n, N}\right)\left\|S_{N} u_{n}-y_{n, N-1}\right\|^{2} \\
& +\lambda \alpha_{n}\|p\|\left(2\left\|y_{n, N}-p\right\|+\lambda \alpha_{n}\|p\|\right)
\end{aligned}
$$

$$
\begin{aligned}
\leq & \beta_{n}\left\|Q y_{n, N}-p\right\|^{2}+\left\|x_{n}-p\right\|^{2} \\
& -\beta_{n, N}\left(1-\beta_{n, N}\right)\left\|S_{N} u_{n}-y_{n, N-1}\right\|^{2} \\
& +\lambda \alpha_{n}\|p\|\left(2\left\|y_{n, N}-p\right\|+\lambda \alpha_{n}\|p\|\right) .
\end{aligned}
$$

So, we have

$$
\begin{aligned}
\beta_{n, N} & \left(1-\beta_{n, N}\right)\left\|S_{N} u_{n}-y_{n, N-1}\right\|^{2} \\
\leq & \beta_{n}\left\|Q y_{n, N}-p\right\|^{2}+\left\|x_{n}-p\right\|^{2}-\left\|y_{n}-p\right\|^{2} \\
& +\lambda \alpha_{n}\|p\|\left(2\left\|y_{n, N}-p\right\|+\lambda \alpha_{n}\|p\|\right) \\
\leq & \beta_{n}\left\|Q y_{n, N}-p\right\|^{2}+\left(\left\|x_{n}-p\right\|+\left\|y_{n}-p\right\|\right)\left\|x_{n}-y_{n}\right\| \\
& +\lambda \alpha_{n}\|p\|\left(2\left\|y_{n, N}-p\right\|+\lambda \alpha_{n}\|p\|\right) .
\end{aligned}
$$

Since $\alpha_{n} \rightarrow 0, \beta_{n} \rightarrow 0,0<\liminf _{n \rightarrow \infty} \beta_{n, N} \leq$ $\lim \sup _{n \rightarrow \infty} \beta_{n, N}<1$, and $\lim _{n \rightarrow \infty}\left\|x_{n}-y_{n}\right\|=0$, it is known that $\left\{\left\|S_{N} u_{n}-y_{n, N-1}\right\|\right\}$ is a null sequence.

Let $i \in\{1, \ldots, N-1\}$. Then, one has

$$
\begin{aligned}
\| y_{n}- & p \|^{2} \\
\leq & \beta_{n}\left\|Q y_{n, N}-p\right\|^{2}+\left\|\tilde{y}_{n, N}-p\right\|^{2} \\
\leq & \beta_{n}\left\|Q y_{n, N}-p\right\|^{2}+\left(\left\|y_{n, N}-p\right\|+\lambda \alpha_{n}\|p\|\right)^{2} \\
= & \beta_{n}\left\|Q y_{n, N}-p\right\|^{2}+\left\|y_{n, N}-p\right\|^{2} \\
& +\lambda \alpha_{n}\|p\|\left(2\left\|y_{n, N}-p\right\|+\lambda \alpha_{n}\|p\|\right) \\
\leq & \beta_{n}\left\|Q y_{n, N}-p\right\|^{2}+\beta_{n, N}\left\|S_{N} u_{n}-p\right\|^{2} \\
& +\left(1-\beta_{n, N}\right)\left\|y_{n, N-1}-p\right\|^{2} \\
& +\lambda \alpha_{n}\|p\|\left(2\left\|y_{n, N}-p\right\|+\lambda \alpha_{n}\|p\|\right) \\
\leq & \beta_{n}\left\|Q y_{n, N}-p\right\|^{2}+\beta_{n, N}\left\|x_{n}-p\right\|^{2} \\
& +\left(1-\beta_{n, N}\right)\left\|y_{n, N-1}-p\right\|^{2} \\
& +\lambda \alpha_{n}\|p\|\left(2\left\|y_{n, N}-p\right\|+\lambda \alpha_{n}\|p\|\right) \\
\leq & \beta_{n}\left\|Q y_{n, N}-p\right\|^{2}+\lambda \alpha_{n}\|p\|\left(2\left\|y_{n, N}-p\right\|+\lambda \alpha_{n}\|p\|\right) \\
& +\beta_{n, N}\left\|x_{n}-p\right\|^{2} \\
& +\left(1-\beta_{n, N}\right)\left[\beta_{n, N-1}\left\|S_{N-1} u_{n}-p\right\|^{2}\right. \\
\leq & \beta_{n}\left\|Q y_{n, N}-p\right\|^{2}+\lambda \alpha_{n}\|p\|\left(2\left\|y_{n, N}-p\right\|+\lambda \alpha_{n}\|p\|\right)
\end{aligned}
$$




$$
\begin{aligned}
& +\left(\beta_{n, N}+\left(1-\beta_{n, N}\right) \beta_{n, N-1}\right)\left\|x_{n}-p\right\|^{2} \\
& +\prod_{k=N-1}^{N}\left(1-\beta_{n, k}\right)\left\|y_{n, N-2}-p\right\|^{2},
\end{aligned}
$$

and so, after $(N-i+1)$ iterations,

$$
\begin{aligned}
\| y_{n}- & p \|^{2} \\
\leq & \beta_{n}\left\|Q y_{n, N}-p\right\|^{2}+\lambda \alpha_{n}\|p\|\left(2\left\|y_{n, N}-p\right\|+\lambda \alpha_{n}\|p\|\right) \\
& +\left(\beta_{n, N}+\sum_{j=i+2}^{N}\left(\prod_{l=j}^{N}\left(1-\beta_{n, l}\right)\right) \beta_{n, j-1}\right) \\
& \times\left\|x_{n}-p\right\|^{2}+\prod_{k=i+1}^{N}\left(1-\beta_{n, k}\right)\left\|y_{n, i}-p\right\|^{2} \\
\leq & \beta_{n}\left\|Q y_{n, N}-p\right\|^{2}+\lambda \alpha_{n}\|p\|\left(2\left\|y_{n, N}-p\right\|+\lambda \alpha_{n}\|p\|\right) \\
& +\left(\beta_{n, N}+\sum_{j=i+2}^{N}\left(\prod_{l=j}^{N}\left(1-\beta_{n, l}\right)\right) \beta_{n, j-1}\right) \\
& \times\left\|x_{n}-p\right\|^{2}+\prod_{k=i+1}^{N}\left(1-\beta_{n, k}\right) \\
& \times\left[\beta_{n, i}\left\|S_{i} u_{n}-p\right\|^{2}+\left(1-\beta_{n, i}\right)\left\|y_{n, i-1}-p\right\|^{2}\right. \\
& \left.-\beta_{n, i}\left(1-\beta_{n, i}\right)\left\|S_{i} u_{n}-y_{n, i-1}\right\|^{2}\right] \\
\leq & \beta_{n}\left\|Q y_{n, N}-p\right\|^{2}+\lambda \alpha_{n}\|p\|\left(2\left\|y_{n, N}-p\right\|+\lambda \alpha_{n}\|p\|\right) \\
& +\left\|x_{n}-p\right\|^{2}-\beta_{n, i} \prod_{k=i}^{N}\left(1-\beta_{n, k}\right)\left\|S_{i} u_{n}-y_{n, i-1}\right\|^{2} .
\end{aligned}
$$

Again, we obtain that

$$
\begin{aligned}
\beta_{n, i} \prod_{k=i}^{N}\left(1-\beta_{n, k}\right)\left\|S_{i} u_{n}-y_{n, i-1}\right\|^{2} \\
\leq \beta_{n}\left\|Q y_{n, N}-p\right\|^{2}+\lambda \alpha_{n}\|p\|\left(2\left\|y_{n, N}-p\right\|+\lambda \alpha_{n}\|p\|\right) \\
\quad+\left\|x_{n}-p\right\|^{2}-\left\|y_{n}-p\right\|^{2} \\
\leq \beta_{n}\left\|Q y_{n, N}-p\right\|^{2}+\lambda \alpha_{n}\|p\|\left(2\left\|y_{n, N}-p\right\|+\lambda \alpha_{n}\|p\|\right) \\
\quad+\left(\left\|x_{n}-p\right\|+\left\|y_{n}-p\right\|\right)\left\|x_{n}-y_{n}\right\| .
\end{aligned}
$$

Since $\alpha_{n} \rightarrow 0, \beta_{n} \rightarrow 0,0<\liminf _{n \rightarrow \infty} \beta_{n, i} \leq$ $\lim \sup _{n \rightarrow \infty} \beta_{n, i}<1$ for each $i=1, \ldots, N-1$, and $\lim _{n \rightarrow \infty}\left\|x_{n}-y_{n}\right\|=0$, it is known that

$$
\lim _{n \rightarrow \infty}\left\|S_{i} u_{n}-y_{n, i-1}\right\|=0 .
$$

Obviously, for $i=1$, we have $\left\|S_{1} u_{n}-u_{n}\right\| \rightarrow 0$.
To conclude, we have that

$$
\begin{aligned}
\left\|S_{2} u_{n}-u_{n}\right\| & \leq\left\|S_{2} u_{n}-y_{n, 1}\right\|+\left\|y_{n, 1}-u_{n}\right\| \\
& =\left\|S_{2} u_{n}-y_{n, 1}\right\|+\beta_{n, 1}\left\|S_{1} u_{n}-u_{n}\right\|,
\end{aligned}
$$

from which $\left\|S_{2} u_{n}-u_{n}\right\| \rightarrow 0$. Thus, by induction $\| S_{i} u_{n}-$ $u_{n} \| \rightarrow 0$ for all $i=2, \ldots, N$ since it is enough to observe that

$$
\begin{aligned}
\left\|S_{i} u_{n}-u_{n}\right\| & \\
\leq & \left\|S_{i} u_{n}-y_{n, i-1}\right\|+\left\|y_{n, i-1}-S_{i-1} u_{n}\right\|+\left\|S_{i-1} u_{n}-u_{n}\right\| \\
\leq & \left\|S_{i} u_{n}-y_{n, i-1}\right\|+\left(1-\beta_{n, i-1}\right)\left\|S_{i-1} u_{n}-y_{n, i-2}\right\| \\
& +\left\|S_{i-1} u_{n}-u_{n}\right\| .
\end{aligned}
$$

Remark 22. As an example, we consider $N=2$ and the following sequences:
(a) $\sigma_{n}=1 / 2+2 / n, \gamma_{n}=\delta_{n}=1 / 4-1 / n$ for all $n>4$;
(b) $\beta_{n}=1 / \sqrt{n}, r_{n}=2-1 / n$, for all $n>1$;
(c) $\beta_{n, 1}=1 / 2-1 / n, \beta_{n, 2}=1 / 2-1 / n^{2}$, for all $n>2$.

Then, they satisfy the hypotheses on the parameter sequences in Lemma 21.

Lemma 23. Let one suppose that $\Omega \neq \emptyset$ and $\beta_{n, i} \rightarrow \beta_{i}$ for all $i$ as $n \rightarrow \infty$. Suppose there exists $k \in\{1, \ldots, N\}$ such that $\beta_{n, k} \rightarrow 0$ as $n \rightarrow \infty$. Let $k_{0} \in\{1, \ldots, N\}$ be the largest index such that $\beta_{n, k_{0}} \rightarrow 0$ as $n \rightarrow \infty$. Suppose that

(i) $\left(\alpha_{n}+\beta_{n}\right) / \beta_{n, k_{0}} \rightarrow 0$ as $n \rightarrow \infty$;

(ii) if $i \leq k_{0}$ and $\beta_{n, i} \rightarrow 0$, then $\beta_{n, k_{0}} / \beta_{n, i} \rightarrow 0$ as $n \rightarrow$ $\infty$;

(iii) if $\beta_{n, i} \rightarrow \beta_{i} \neq 0$, then $\beta_{i}$ lies in $(0,1)$.

Moreover, suppose that (H1), (H7), and (H8) hold. Then, $\lim _{n \rightarrow \infty}\left\|S_{i} u_{n}-u_{n}\right\|=0$ for each $i=1, \ldots, N$.

Proof. First of all, we note that if (H7) holds, then also (H2)(H6) are satisfied. So $\left\{x_{n}\right\}$ is asymptotically regular. Let $k_{0}$ be as in the hypotheses. As in Lemma 21, for every index $i \in\{1, \ldots, N\}$ such that $\beta_{n, i} \rightarrow \beta_{i} \neq 0$ (which leads to $\left.0<\liminf _{n \rightarrow \infty} \beta_{n, i} \leq \lim \sup _{n \rightarrow \infty} \beta_{n, i}<1\right)$, one has $\| S_{i} u_{n}-$ $y_{n, i-1} \| \rightarrow 0$ as $n \rightarrow \infty$.

For all the other indexes $i \leq k_{0}$, we can prove that $\| S_{i} u_{n}-$ $y_{n, i-1} \| \rightarrow 0$ as $n \rightarrow \infty$ in a similar manner. By the following relation (due to (86)):

$$
\begin{aligned}
& \left\|x_{n+1}-p\right\|^{2} \\
& \quad \leq \sigma_{n}\left\|y_{n}-p\right\|^{2}+\left(\gamma_{n}+\delta_{n}\right)\left\|\tilde{y}_{n}-p\right\|^{2} \\
& \quad=\sigma_{n}\left\|y_{n}-p\right\|^{2}+\left(1-\sigma_{n}\right)\left\|\tilde{y}_{n}-p\right\|^{2}
\end{aligned}
$$




$$
\begin{aligned}
\leq & \sigma_{n}\left\|y_{n}-p\right\|^{2}+\left(1-\sigma_{n}\right)\left(\left\|y_{n}-p\right\|+\lambda \alpha_{n}\|p\|^{2}\right. \\
\leq & \left(\left\|y_{n}-p\right\|+\lambda \alpha_{n}\|p\|\right)^{2} \\
= & \left\|y_{n}-p\right\|^{2}+\lambda \alpha_{n}\|p\|\left(2\left\|y_{n}-p\right\|+\lambda \alpha_{n}\|p\|\right) \\
\leq & \beta_{n}\left\|Q y_{n, N}-p\right\|^{2}+\lambda \alpha_{n}\|p\|\left(2\left\|y_{n, N}-p\right\|+\lambda \alpha_{n}\|p\|\right) \\
& +\left\|x_{n}-p\right\|^{2}-\beta_{n, i} \prod_{k=i}^{N}\left(1-\beta_{n, k}\right)\left\|S_{i} u_{n}-y_{n, i-1}\right\|^{2} \\
& +\lambda \alpha_{n}\|p\|\left(2\left\|y_{n}-p\right\|+\lambda \alpha_{n}\|p\|\right) \\
\leq & \beta_{n}\left\|Q y_{n, N}-p\right\|^{2}+2 \lambda \alpha_{n}\|p\| \\
& \times\left(\left\|x_{n}-p\right\|+\left\|y_{n}-p\right\|+\lambda \alpha_{n}\|p\|\right) \\
& +\left\|x_{n}-p\right\|^{2}-\beta_{n, i} \prod_{k=i}^{N}\left(1-\beta_{n, k}\right)\left\|S_{i} u_{n}-y_{n, i-1}\right\|^{2},
\end{aligned}
$$

we immediately obtain that

$$
\begin{aligned}
\prod_{k=i}^{N}(1 & \left.-\beta_{n, k}\right)\left\|S_{i} u_{n}-y_{n, i-1}\right\|^{2} \\
\leq & \frac{\beta_{n}}{\beta_{n, i}}\left\|Q y_{n, N}-p\right\|^{2}+\frac{\alpha_{n}}{\beta_{n, i}} 2 \lambda\|p\| \\
& \times\left(\left\|x_{n}-p\right\|+\left\|y_{n}-p\right\|+\lambda \alpha_{n}\|p\|\right) \\
& +\left(\left\|x_{n}-p\right\|+\left\|x_{n+1}-p\right\|\right) \frac{\left\|x_{n}-x_{n+1}\right\|}{\beta_{n, i}} .
\end{aligned}
$$

By Lemma 19 or by hypothesis (ii) of the sequences, we have

$$
\frac{\left\|x_{n}-x_{n+1}\right\|}{\beta_{n, i}}=\frac{\left\|x_{n}-x_{n+1}\right\|}{\beta_{n, k_{0}}} \cdot \frac{\beta_{n, k_{0}}}{\beta_{n, i}} \longrightarrow 0 .
$$

So, the thesis follows.

Remark 24. Let us consider $N=3$ and the following sequences:

(a) $\alpha_{n}=1 / n^{5 / 4}, \beta_{n}=1 / n^{1 / 2}, r_{n}=2-1 / n^{2}$, for all $n>1$;

(b) $\sigma_{n}=1 / 2+2 / n^{2}, \gamma_{n}=\delta_{n}=1 / 4-1 / n^{2}$, for all $n>2$;

(c) $\beta_{n, 1}=1 / n^{1 / 4}, \beta_{n, 2}=1 / 2-1 / n^{2}, \beta_{n, 3}=1 / n^{1 / 3}$, for all $n>1$.

It is easy to see that all hypotheses (i)-(iii), (H1), (H7), and (H8) of Lemma 23 are satisfied.

Remark 25. Under the hypotheses of Lemma 23, analogously to Lemma 21, one can see that

$$
\lim _{n \rightarrow \infty}\left\|S_{i} u_{n}-y_{n, i-1}\right\|=0, \quad \forall i \in\{2, \ldots, N\} .
$$

Corollary 26. Let one suppose that the hypotheses of either Lemma 21 or Lemma 23 are satisfied. Then, $\omega_{w}\left(x_{n}\right)=$ $\omega_{w}\left(u_{n}\right)=\omega_{w}\left(y_{n, 1}\right), \omega_{s}\left(x_{n}\right)=\omega_{s}\left(u_{n}\right)=\omega_{s}\left(y_{n, 1}\right)$, and $\omega_{w}\left(x_{n}\right) \subset \Omega$.
Proof. By Remark 18, we have $\omega_{w}\left(x_{n}\right)=\omega_{w}\left(u_{n}\right)$ and $\omega_{s}\left(x_{n}\right)=$ $\omega_{s}\left(u_{n}\right)$. Observe that

$$
\begin{aligned}
\left\|x_{n}-y_{n, 1}\right\| & \leq\left\|x_{n}-u_{n}\right\|+\left\|y_{n, 1}-u_{n}\right\| \\
& =\left\|x_{n}-u_{n}\right\|+\beta_{n, 1}\left\|S_{1} u_{n}-u_{n}\right\| .
\end{aligned}
$$

By Lemmas 17 and 21, $\left\|x_{n}-u_{n}\right\| \rightarrow 0$ and $\left\|S_{1} u_{n}-u_{n}\right\| \rightarrow 0$ as $n \rightarrow \infty$, and hence

$$
\lim _{n \rightarrow \infty}\left\|x_{n}-y_{n, 1}\right\|=0
$$

So, we get $\omega_{w}\left(x_{n}\right)=\omega_{w}\left(y_{n, 1}\right)$ and $\omega_{s}\left(x_{n}\right)=\omega_{s}\left(y_{n, 1}\right)$.

Let $p \in \omega_{w}\left(x_{n}\right)$. Since $p \in \omega_{w}\left(u_{n}\right)$, by Lemma 21 and Lemma 7(ii) (demiclosedness principle), we have $p \in$ $\operatorname{Fix}\left(S_{i}\right)$ for all index $i$, that is, $p \in \bigcap_{i} \operatorname{Fix}\left(S_{i}\right)$. Taking into consideration that $T$ is $\zeta$-strictly pseudocontractive, by Lemma 7(i), we get

$$
\begin{aligned}
& \left\|T x_{n}-x_{n}\right\| \\
& \quad \leq\left\|T x_{n}-T y_{n}\right\|+\left\|T y_{n}-y_{n}\right\|+\left\|y_{n}-x_{n}\right\| \\
& \quad \leq \frac{1+\zeta}{1-\zeta}\left\|x_{n}-y_{n}\right\|+\left\|y_{n}-T y_{n}\right\|+\left\|x_{n}-y_{n}\right\| \\
& \quad=\frac{2}{1-\zeta}\left\|x_{n}-y_{n}\right\|+\left\|y_{n}-T y_{n}\right\|,
\end{aligned}
$$

which together with $\left\|x_{n}-y_{n}\right\| \rightarrow 0$ (by Lemma 17) and $\| y_{n}-$ $T y_{n} \| \rightarrow 0$ (by (82)) implies that

$$
\lim _{n \rightarrow \infty}\left\|x_{n}-T x_{n}\right\|=0 .
$$

Utilizing Lemma 7(ii) (demiclosedness principle), we have $p \in \operatorname{Fix}(T)$. Furthermore, by Lemmas 14 and 17, we know that $p \in \operatorname{EP}(F, h)$. Finally, by similar argument as in [18], we can show that $p \in \Gamma$, and as a result $p \in \Omega$.

Theorem 27. Let one suppose that $\Omega \neq \emptyset$. Let $\left\{\beta_{n}\right\},\left\{\beta_{n, i}\right\}, i=$ $1, \ldots, N$, be sequences in $(0,1)$ such that $0<\liminf _{n \rightarrow \infty} \beta_{n, i} \leq$ $\limsup _{n \rightarrow \infty} \beta_{n, i}<1$ for all index $i$. Moreover, Let one suppose that (H1)-(H6) hold. Then, the sequences $\left\{x_{n}\right\},\left\{y_{n}\right\}$, and $\left\{u_{n}\right\}$, explicitly defined by scheme (30), all converge strongly to the unique solution $x^{*} \in \Omega$ of the following variational inequality:

$$
\left\langle Q x^{*}-x^{*}, z-x^{*}\right\rangle \leq 0, \quad \forall z \in \Omega \text {. }
$$

Proof. Since the mapping $P_{\Omega} Q$ is a $\rho$-contraction, it has a unique fixed point $x^{*}$; it is the unique solution of (99). Since (H1)-(H6) hold, the sequence $\left\{x_{n}\right\}$ is asymptotically regular (by Lemma 16). In terms of Lemma 17, $\left\|x_{n}-y_{n}\right\| \rightarrow 0$ and 
$\left\|x_{n}-u_{n}\right\| \rightarrow 0$ as $n \rightarrow \infty$. Moreover, utilizing Lemmas 8 and 10, we have from (33)-(34) the following:

$$
\begin{aligned}
& \left\|x_{n+1}-x^{*}\right\|^{2} \\
& \leq \sigma_{n}\left\|y_{n}-x^{*}\right\|^{2}+\left(1-\sigma_{n}\right)\left\|\tilde{y}_{n}-x^{*}\right\|^{2} \\
& \leq \sigma_{n}\left\|y_{n}-x^{*}\right\|^{2}+\left(1-\sigma_{n}\right)\left(\left\|y_{n}-x^{*}\right\|+\lambda \alpha_{n}\|p\|\right)^{2} \\
& \leq\left(\left\|y_{n}-x^{*}\right\|+\lambda \alpha_{n}\|p\|\right)^{2} \\
& =\left\|y_{n}-x^{*}\right\|^{2}+\lambda \alpha_{n}\|p\|\left(2\left\|y_{n}-x^{*}\right\|+\lambda \alpha_{n}\|p\|\right) \\
& \leq\left\|\beta_{n}\left(Q y_{n, N}-Q x^{*}\right)+\left(1-\beta_{n}\right)\left(\tilde{y}_{n, N}-x^{*}\right)\right\|^{2} \\
& +2 \beta_{n}\left\langle Q x^{*}-x^{*}, y_{n}-x^{*}\right\rangle \\
& +\lambda \alpha_{n}\|p\|\left(2\left\|y_{n}-x^{*}\right\|+\lambda \alpha_{n}\|p\|\right) \\
& \leq\left(\beta_{n} \rho\left\|y_{n, N}-x^{*}\right\|+\left(1-\beta_{n}\right)\left\|y_{n, N}-x^{*}\right\|\right)^{2} \\
& +2 \beta_{n}\left\langle Q x^{*}-x^{*}, y_{n}-x^{*}\right\rangle \\
& +\lambda \alpha_{n}\|p\|\left(2\left\|y_{n}-x^{*}\right\|+\lambda \alpha_{n}\|p\|\right) \\
& =\left(1-(1-\rho) \beta_{n}\right)^{2}\left\|y_{n, N}-x^{*}\right\|^{2} \\
& +2 \beta_{n}\left\langle Q x^{*}-x^{*}, y_{n}-x^{*}\right\rangle \\
& +\lambda \alpha_{n}\|p\|\left(2\left\|y_{n}-x^{*}\right\|+\lambda \alpha_{n}\|p\|\right) \\
& \leq\left(1-(1-\rho) \beta_{n}\right)\left\|u_{n}-x^{*}\right\|^{2} \\
& +2 \beta_{n}\left\langle Q x^{*}-x^{*}, y_{n}-x^{*}\right\rangle \\
& +\lambda \alpha_{n}\|p\|\left(2\left\|y_{n}-x^{*}\right\|+\lambda \alpha_{n}\|p\|\right) \\
& \leq\left(1-(1-\rho) \beta_{n}\right)\left\|x_{n}-x^{*}\right\|^{2} \\
& +2 \beta_{n}\left\langle Q x^{*}-x^{*}, y_{n}-x^{*}\right\rangle \\
& +\lambda \alpha_{n}\|p\|\left(2\left\|y_{n}-x^{*}\right\|+\lambda \alpha_{n}\|p\|\right) \\
& =\left(1-(1-\rho) \beta_{n}\right)\left\|x_{n}-x^{*}\right\|^{2} \\
& +(1-\rho) \beta_{n} \cdot \frac{2}{1-\rho}\left\langle Q x^{*}-x^{*}, y_{n}-x^{*}\right\rangle \\
& +\lambda \alpha_{n}\|p\|\left(2\left\|y_{n}-x^{*}\right\|+\lambda \alpha_{n}\|p\|\right) .
\end{aligned}
$$

Now, let $\left\{x_{n_{k}}\right\}$ be a subsequence of $\left\{x_{n}\right\}$ such that

$$
\limsup _{n \rightarrow \infty}\left\langle Q x^{*}-x^{*}, x_{n}-x^{*}\right\rangle=\lim _{k \rightarrow \infty}\left\langle Q x^{*}-x^{*}, x_{n_{k}}-x^{*}\right\rangle .
$$

By the boundedness of $\left\{x_{n}\right\}$, we may assume, without loss of generality, that $x_{n_{k}} \rightarrow z \in \omega_{w}\left(x_{n}\right)$. According to Corollary 26, we know that $\omega_{w}\left(x_{n}\right) \subset \Omega$, and hence $z \in \Omega$. Taking into consideration that $x^{*}=P_{\Omega} Q x^{*}$ we obtain from (101) that

$$
\begin{aligned}
& \limsup _{n \rightarrow \infty}\left\langle Q x^{*}-x^{*}, y_{n}-x^{*}\right\rangle \\
& \quad=\limsup _{n \rightarrow \infty}\left[\left\langle Q x^{*}-x^{*}, x_{n}-x^{*}\right\rangle+\left\langle Q x^{*}-x^{*}, y_{n}-x_{n}\right\rangle\right] \\
& \quad=\limsup _{n \rightarrow \infty}\left\langle Q x^{*}-x^{*}, x_{n}-x^{*}\right\rangle \\
& \quad=\lim _{k \rightarrow \infty}\left\langle Q x^{*}-x^{*}, x_{n_{k}}-x^{*}\right\rangle \\
& =\left\langle Q x^{*}-x^{*}, z-x^{*}\right\rangle \leq 0 .
\end{aligned}
$$

Since $\sum_{n=0}^{\infty} \alpha_{n}<\infty$ and $\sum_{n=0}^{\infty} \beta_{n}=\infty$, we deduce that $\sum_{n=0}^{\infty} \lambda \alpha_{n}\|p\|\left(2\left\|y_{n}-x^{*}\right\|+\lambda \alpha_{n}\|p\|\right)<\infty$ and $\sum_{n=0}^{\infty}(1-\rho) \beta_{n}=$ $\infty$. In terms of Lemma 9 we derive $x_{n} \rightarrow x^{*}$ as $n \rightarrow \infty$.

In a similar way, we can derive the following result.

Theorem 28. Let one suppose that $\Omega \neq \emptyset$. Let $\left\{\beta_{n}\right\},\left\{\beta_{n, i}\right\}, i=$ $1, \ldots, N$, be sequences in $(0,1)$ such that $\beta_{n, i} \rightarrow \beta_{i}$ for all $i$ as $n \rightarrow \infty$. Suppose that there exists $k \in\{1, \ldots, N\}$ for which $\beta_{n, k} \rightarrow 0$ as $n \rightarrow \infty$. Let $k_{0} \in\{1, \ldots, N\}$ the largest index for which $\beta_{n, k_{0}} \rightarrow 0$. Moreover, let one suppose that (H1), (H7), and (H8) hold, and

(i) $\left(\alpha_{n}+\beta_{n}\right) / \beta_{n, k_{0}} \rightarrow 0$ as $n \rightarrow \infty$;

(ii) if $i \leq k_{0}$ and $\beta_{n, i} \rightarrow 0$, then $\beta_{n, k_{0}} / \beta_{n, i} \rightarrow 0$ as $n \rightarrow$ $\infty$;

(iii) if $\beta_{n, i} \rightarrow \beta_{i} \neq 0$, then $\beta_{i}$ lies in $(0,1)$.

Then, the sequences $\left\{x_{n}\right\},\left\{y_{n}\right\}$, and $\left\{u_{n}\right\}$ explicitly defined by scheme (30) all converge strongly to the unique solution $x^{*} \in \Omega$ of the following variational inequality:

$$
\left\langle Q x^{*}-x^{*}, z-x^{*}\right\rangle \leq 0, \quad \forall z \in \Omega .
$$

Remark 29. According to the above argument processes for Theorems 27 and 28, we can readily see that if in scheme (30), the iterative step $y_{n}=\beta_{n} Q y_{n, N}+\left(1-\beta_{n}\right) P_{C}\left(y_{n, N}-\lambda \nabla f_{\alpha_{n}}\left(y_{n, N}\right)\right)$ is replaced by the iterative one $y_{n}=\beta_{n} Q x_{n}+\left(1-\beta_{n}\right) P_{C}\left(y_{n, N}-\right.$ $\left.\lambda \nabla f_{\alpha_{n}}\left(y_{n, N}\right)\right)$, then Theorems 27 and 28 remain valid.

Remark 30. Theorems 27 and 28 improve, extend, supplement, and develop [17, Theorems 3.12 and 3.13] and [1, Theorems 5.2 and 6.1] in the following aspects:

(a) the multistep iterative scheme (30) of [17] is extended to develop our relaxed viscosity iterative scheme (30) with regularization for MP (3), EP (10), and strict pseudocontraction $T$ by virtue of Xu iterative schemes in $[1]$;

(b) the argument techniques in Theorems 27 and 28 are very different from the ones in [17, Theorems 3.12 and 3.13] and the ones in [1, Theorems 5.2 and 6.1] because we use the properties of strict pseudocontractive mappings and maximal monotone mappings (see, e.g., Lemmas 7 and 10); 
(c) compared with the proof of Theorems 5.2 and 6.1 in [1], the proof of Theorems 27 and 28 shows that $\lim _{n \rightarrow \infty}\left\|y_{n, N}-P_{C}\left(I-\lambda \nabla f_{\alpha_{n}}\right) y_{n, N}\right\|=\lim _{n \rightarrow \infty} \| y_{n}-$ $P_{C}\left(I-\lambda \nabla f_{\alpha_{n}}\right) y_{n} \|=0$ via the argument of $\lim _{n \rightarrow \infty}\left\|\nabla f_{\alpha_{n}}\left(y_{n, N}\right)-\nabla f(p)\right\|=0$, for all $p \in \Omega$ (see Lemma 20 and its proof);

(d) the problem of finding an element of $\operatorname{Fix}(T) \cap$ $\left(\bigcap_{i} \operatorname{Fix}\left(S_{i}\right)\right) \cap \operatorname{EP}(F, h) \cap \Gamma$ in Theorems 27 and 28 is more general than the one of finding an element of $\operatorname{Fix}(T) \cap\left(\bigcap_{i} \operatorname{Fix}\left(S_{i}\right)\right) \cap \operatorname{EP}(F, h)$ in [17, Theorems 3.12 and 3.13] and the one of finding an element of $\Gamma$ in $[1$, Theorems 5.2 and 6.1].

\section{Applications}

For a given nonlinear mapping $A: C \rightarrow H$, we consider again the variational inequality problem (VIP) of finding $\bar{x} \epsilon$ $C$ such that

$$
\langle A \bar{x}, y-\bar{x}\rangle \geq 0, \quad \forall y \in C
$$
holds:

Recall that if $u$ is a point in $C$, then the following relation

$$
u \in \operatorname{VI}(C, A) \Longleftrightarrow u=P_{C}(I-\lambda A) u, \quad \text { for some } \lambda>0
$$

from which we have the following relation:

$$
\begin{array}{r}
u \in \Gamma \Longleftrightarrow u \in \mathrm{VI}(C, \nabla f) \Longleftrightarrow u=P_{C}(I-\lambda \nabla f) u, \\
\text { for some } \lambda>0 .
\end{array}
$$

An operator $A: C \rightarrow H$ is said to be an $\alpha$-inverse strongly monotone operator if there exists a constant $\alpha>0$ such that

$$
\langle A x-A y, x-y\rangle \geq \alpha\|A x-A y\|^{2}, \quad \forall x, y \in C .
$$

As an example, we recall that the $\alpha$-inverse strongly monotone operators are firmly nonexpansive mappings if $\alpha \geq$ 1 and that every $\alpha$-inverse strongly monotone operator is also a $(1 / \alpha)$ Lipschitz continuous (see [19]). We observe that, if $A$ is $\alpha$-inverse strongly monotone, the mapping $P_{C}(I-\mu A)$ is nonexpansive for all $\mu \in(0,2 \alpha]$ since they are compositions of nonexpansive mappings (see [19, page 419]).

Let us consider $\widetilde{S}_{1}, \ldots, \widetilde{S}_{M}$ a finite number of nonexpansive self-mappings on $C$ and $A_{1}, \ldots, A_{N}$ be a finite number of $\alpha$-inverse strongly monotone operators. Let $T: C \rightarrow C$ be a $\zeta$-strict pseudocontraction on $C$ with fixed points. Let us consider the following mixed problem of finding $x^{*} \epsilon$ $\operatorname{Fix}(T) \cap \operatorname{EP}(F, h) \cap \Gamma$ such that

$$
\begin{gathered}
\left\langle\left(I-\widetilde{S}_{1}\right) x^{*}, y-x^{*}\right\rangle \geq 0, \quad \forall y \in \operatorname{Fix}(T) \cap \operatorname{EP}(F, h) \cap \Gamma, \\
\left\langle\left(I-\widetilde{S}_{2}\right) x^{*}, y-x^{*}\right\rangle \geq 0, \quad \forall y \in \operatorname{Fix}(T) \cap \operatorname{EP}(F, h) \cap \Gamma, \\
\vdots \\
\left\langle\left(I-\widetilde{S}_{M}\right) x^{*}, y-x^{*}\right\rangle \geq 0, \quad \forall y \in \operatorname{Fix}(T) \cap \operatorname{EP}(F, h) \cap \Gamma, \\
\left\langle A_{1} x^{*}, y-x^{*}\right\rangle \geq 0, \quad \forall y \in C, \\
\left\langle A_{2} x^{*}, y-x^{*}\right\rangle \geq 0, \quad \forall y \in C, \\
\vdots \\
\left\langle A_{N} x^{*}, y-x^{*}\right\rangle \geq 0, \quad \forall y \in C .
\end{gathered}
$$

We denote by (SVI) the set of solutions of the above $(N+$ $M)$ system. This problem is equivalent to finding a common fixed point of $T,\left\{P_{\mathrm{Fix}(T) \cap \mathrm{EP}(F, h) \cap \Gamma} \widetilde{S}_{i}\right\}_{i=1}^{N},\left\{P_{C}\left(I-\mu A_{i}\right)\right\}_{i=1}^{M}$. The following results are then consequences of Theorems 27 and 28.

Theorem 31. Let one suppose that $\Omega=\operatorname{Fix}(T) \cap(\mathrm{SVI}) \cap$ $\mathrm{EP}(\mathrm{F}, \mathrm{h}) \cap \Gamma \neq \emptyset$. Fix $\mu \in(0,2 \alpha]$, and $\lambda \in(0,2 / L)$. Let $\left\{\alpha_{n}\right\}$, $\left\{\beta_{n, i}\right\}, i=1, \ldots,(M+N)$, be sequences in $(0,1)$ such that $0<\liminf _{n \rightarrow \infty} \beta_{n, i} \leq \limsup _{n \rightarrow \infty} \beta_{n, i}<1$ for all index i. Moreover, Let one suppose that (H1)-(H6) hold. Then the sequences $\left\{x_{n}\right\},\left\{y_{n}\right\}$, and $\left\{u_{n}\right\}$ explicitly defined by the following scheme:

$$
\begin{aligned}
F\left(u_{n}, y\right)+h\left(u_{n}, y\right)+\frac{1}{r_{n}}\left\langle y-u_{n}, u_{n}-x_{n}\right\rangle \geq 0, \quad \forall y \in C, & \beta_{n, 1} P_{\operatorname{Fix}(T) \cap \operatorname{EP}(F, h) \cap \Gamma} \widetilde{S}_{1} u_{n}+\left(1-\beta_{n, 1}\right) u_{n}, \\
y_{n, i}= & \beta_{n, i} P_{\operatorname{Fix}(T) \cap \mathrm{EP}(F, h) \cap \Gamma} \widetilde{S}_{i} u_{n} \\
& +\left(1-\beta_{n, i}\right) y_{n, i-1}, \quad i=2, \ldots, M, \\
y_{n, M+j}= & \beta_{n, M+j} P_{C}\left(I-\mu A_{j}\right) u_{n} \\
& +\left(1-\beta_{n, M+j}\right) y_{n, M+j-1}, \quad j=1, \ldots, N, \\
y_{n}= & \beta_{n} Q y_{n, M+N}+\left(1-\beta_{n}\right) \\
& \times P_{C}\left(y_{n, M+N}-\lambda \nabla f_{\alpha_{n}}\left(y_{n, M+N}\right)\right), \\
x_{n+1} & \sigma_{n} y_{n}+\gamma_{n} P_{C}\left(y_{n}-\lambda \nabla f_{\alpha_{n}}\left(y_{n}\right)\right) \\
& +\delta_{n} T P_{C}\left(y_{n}-\lambda \nabla f_{\alpha_{n}}\left(y_{n}\right)\right), \quad \forall n \geq 0
\end{aligned}
$$

all converge strongly to the unique solution $x^{*} \in \Omega$ of the following variational inequality:

$$
\left\langle Q x^{*}-x^{*}, z-x^{*}\right\rangle \leq 0, \quad \forall z \in \Omega .
$$


Theorem 32. Let one suppose that $\Omega \neq \emptyset$. Fix $\mu \in(0,2 \alpha]$ and $\lambda \in(0,2 / L)$. Let $\left\{\beta_{n}\right\},\left\{\beta_{n, i}\right\}, i=1, \ldots,(M+N)$, be sequences in $(0,1)$ and $\beta_{n, i} \rightarrow \beta_{i}$ for all $i$ as $n \rightarrow \infty$. Suppose that there exists $k \in\{1, \ldots, M+N\}$ such that $\beta_{n, k} \rightarrow 0$ as $n \rightarrow \infty$. Let $k_{0} \in\{1, \ldots, M+N\}$ be the largest index for which $\beta_{n, k_{0}} \rightarrow 0$. Moreover, let one suppose that (H1), (H7), and (H8) hold, and

(i) $\left(\alpha_{n}+\beta_{n}\right) / \beta_{n, k_{0}} \rightarrow 0$ as $n \rightarrow \infty$;

(ii) if $i \leq k_{0}$ and $\beta_{n, i} \rightarrow 0$, then $\beta_{n, k_{0}} / \beta_{n, i} \rightarrow 0$ as $n \rightarrow$ $\infty$;

(iii) if $\beta_{n, i} \rightarrow \beta_{i} \neq 0$, then $\beta_{i}$ lies in $(0,1)$.

Then, the sequences $\left\{x_{n}\right\},\left\{y_{n}\right\}$, and $\left\{u_{n}\right\}$ explicitly defined by scheme (109) all converge strongly to the unique solution $x^{*} \in$ $\Omega$ of the following variational inequality:

$$
\left\langle Q x^{*}-x^{*}, z-x^{*}\right\rangle \leq 0, \quad \forall z \in \Omega
$$

Remark 33. If we choose $\nabla f=A_{1}=\cdots=A_{N}=0$ in system (108), we obtain a system of hierarchical fixed point problems introduced by Moudafi and Maingé [20, 21].

Further, utilizing Theorems 27 and 28, we again give the following strong convergence theorems for finding a common element of the solution set $\Gamma$ of MP (3), the solution set $\operatorname{EP}(F, h)$ of $\operatorname{EP}(10)$, and the common fixed point set $\left(\bigcap_{i} \operatorname{Fix}\left(S_{i}\right)\right)$ of a finite family of nonexpansive mappings $S_{i}$ : $C \rightarrow C, i=1, \ldots, N$.

Theorem 34. Let one suppose that $\Omega=\left(\bigcap_{i} \operatorname{Fix}\left(S_{i}\right)\right) \cap$ $\operatorname{EP}(F, h) \cap \Gamma \neq \emptyset$. Let $\left\{\beta_{n}\right\},\left\{\beta_{n, i}\right\}, i=1, \ldots, N$, be sequences in $(0,1)$ such that $0<\liminf _{n \rightarrow \infty} \beta_{n, i} \leq \lim \sup _{n \rightarrow \infty} \beta_{n, i}<1$ for all index $i$. Moreover, Let one suppose that there hold (H1)(H6) with $\gamma_{n}=0$, for all $n \geq 0$. Then, the sequences $\left\{x_{n}\right\},\left\{y_{n}\right\}$, and $\left\{u_{n}\right\}$ generated explicitly by

$$
\begin{gathered}
F\left(u_{n}, y\right)+h\left(u_{n}, y\right)+\frac{1}{r_{n}}\left\langle y-u_{n}, u_{n}-x_{n}\right\rangle \geq 0, \quad \forall y \in C, \\
y_{n, 1}=\beta_{n, 1} S_{1} u_{n}+\left(1-\beta_{n, 1}\right) u_{n}, \\
y_{n, i}=\beta_{n, i} S_{i} u_{n}+\left(1-\beta_{n, i}\right) y_{n, i-1}, \quad i=2, \ldots, N, \\
y_{n}=\beta_{n} Q y_{n, N}+\left(1-\beta_{n}\right) P_{C}\left(y_{n, N}-\lambda \nabla f_{\alpha_{n}}\left(y_{n, N}\right)\right), \\
x_{n+1}=\sigma_{n} y_{n}+\left(1-\sigma_{n}\right) P_{C}\left(y_{n}-\lambda \nabla f_{\alpha_{n}}\left(y_{n}\right)\right), \quad \forall n \geq 0,
\end{gathered}
$$

all converge strongly to the unique solution $x^{*} \in \Omega$ of the following variational inequality:

$$
\left\langle Q x^{*}-x^{*}, z-x^{*}\right\rangle \leq 0, \quad \forall z \in \Omega
$$

Proof. In Theorems 27, put $T=I$ the identity mapping and $\gamma_{n}=0$, for all $n \geq 0$. Then, $T$ is a $\zeta$-strictly pseudocontractive mapping with $\zeta=0$. Hence, we deduce that $\Omega=\operatorname{Fix}(T) \cap$ $\left(\bigcap_{i} \operatorname{Fix}\left(S_{i}\right)\right) \cap \operatorname{EP}(F, h) \cap \Gamma=\left(\bigcap_{i} \operatorname{Fix}\left(S_{i}\right)\right) \cap \operatorname{EP}(F, h) \cap \Gamma \neq \emptyset$, $\left(\gamma_{n}+\delta_{n}\right) \zeta \leq \gamma_{n}$, for all $n \geq 0$, and

$$
\begin{aligned}
x_{n+1}= & \sigma_{n} y_{n}+\gamma_{n} P_{C}\left(y_{n}-\lambda \nabla f_{\alpha_{n}}\left(y_{n}\right)\right) \\
& +\delta_{n} T P_{C}\left(y_{n}-\lambda \nabla f_{\alpha_{n}}\left(y_{n}\right)\right) \\
= & \sigma_{n} y_{n}+\delta_{n} P_{C}\left(y_{n}-\lambda \nabla f_{\alpha_{n}}\left(y_{n}\right)\right) \\
= & \sigma_{n} y_{n}+\left(1-\sigma_{n}\right) P_{C}\left(y_{n}-\lambda \nabla f_{\alpha_{n}}\left(y_{n}\right)\right) .
\end{aligned}
$$

Thus, the conditions in Theorem 27 are all satisfied. and from which we obtain the desired result.

Theorem 35. Let one suppose that $\Omega=\left(\bigcap_{i} \operatorname{Fix}\left(S_{i}\right)\right) \cap$ $\operatorname{EP}(F, h) \cap \Gamma \neq \emptyset$. Let $\left\{\beta_{n}\right\},\left\{\beta_{n, i}\right\}, i=1, \ldots, N$, be sequences in $(0,1)$ such that $\beta_{n, i} \rightarrow \beta_{i}$ for all $i$ as $n \rightarrow \infty$. Suppose that there exists $k \in\{1, \ldots, N\}$ for which $\beta_{n, k} \rightarrow 0$ as $n \rightarrow \infty$. Let $k_{0} \in\{1, \ldots, N\}$ be the largest index for which $\beta_{n, k_{0}} \rightarrow 0$. Moreover, let one suppose that there hold (H1), (H7), and (H8) with $\gamma_{n}=0$, for all $n \geq 0$, and

(i) $\left(\alpha_{n}+\beta_{n}\right) / \beta_{n, k_{0}} \rightarrow 0$ as $n \rightarrow \infty$;

(ii) if $i \leq k_{0}$ and $\beta_{n, i} \rightarrow 0$, then $\beta_{n, k_{0}} / \beta_{n, i} \rightarrow 0$ as $n \rightarrow$ $\infty$;

(iii) if $\beta_{n, i} \rightarrow \beta_{i} \neq 0$, then $\beta_{i}$ lies in $(0,1)$.

Then the sequences $\left\{x_{n}\right\},\left\{y_{n}\right\}$, and $\left\{u_{n}\right\}$ generated explicitly by (112) all converge strongly to the unique solution $x^{*} \in \Omega$ of the following variational inequality:

$$
\left\langle Q x^{*}-x^{*}, z-x^{*}\right\rangle \leq 0, \quad \forall z \in \Omega .
$$

\section{Acknowledgments}

This research was partially supported by the National Science Foundation of China (11071169), Innovation Program of Shanghai Municipal Education Commission (09ZZ133), and Leading Academic Discipline Project of Shanghai Normal University (DZL707). This research was partially supported by the Grant from the NSC 101-2115-M-037-001.

\section{References}

[1] H.-K. Xu, "Averaged mappings and the gradient-projection algorithm," Journal of Optimization Theory and Applications, vol. 150, no. 2, pp. 360-378, 2011.

[2] J.-L. Lions, Quelques Méthodes de Résolution des Problèmes aux Limites non Linéaires, Dunod, Paris, France, 1969.

[3] L. C. Zeng, "Iterative algorithms for finding approximate solutions for general strongly nonlinear variational inequalities," Journal of Mathematical Analysis and Applications, vol. 187, no. 2, pp. 352-360, 1994.

[4] J. C. Yao, "Variational inequalities with generalized monotone operators," Mathematics of Operations Research, vol. 19, no. 3, pp. 691-705, 1994.

[5] L. C. Zeng, S. Schaible, and J. C. Yao, "Iterative algorithm for generalized set-valued strongly nonlinear mixed variationallike inequalities," Journal of Optimization Theory and Applications, vol. 124, no. 3, pp. 725-738, 2005. 
[6] L.-C. Ceng and J.-C. Yao, "An extragradient-like approximation method for variational inequality problems and fixed point problems," Applied Mathematics and Computation, vol. 190, no. 1, pp. 205-215, 2007.

[7] L.-C. Ceng and S. Huang, "Modified extragradient methods for strict pseudo-contractions and monotone mappings," Taiwanese Journal of Mathematics, vol. 13, no. 4, pp. 1197-1211, 2009.

[8] L.-C. Ceng, C.-Y. Wang, and J.-C. Yao, "Strong convergence theorems by a relaxed extragradient method for a general system of variational inequalities," Mathematical Methods of Operations Research, vol. 67, no. 3, pp. 375-390, 2008.

[9] N. Nadezhkina and W. Takahashi, "Weak convergence theorem by an extragradient method for nonexpansive mappings and monotone mappings," Journal of Optimization Theory and Applications, vol. 128, no. 1, pp. 191-201, 2006.

[10] L.-C. Zeng and J.-C. Yao, "Strong convergence theorem by an extragradient method for fixed point problems and variational inequality problems," Taiwanese Journal of Mathematics, vol. 10, no. 5, pp. 1293-1303, 2006.

[11] C. Byrne, "A unified treatment of some iterative algorithms in signal processing and image reconstruction," Inverse Problems, vol. 20, no. 1, pp. 103-120, 2004.

[12] G. Marino and H.-K. Xu, "Weak and strong convergence theorems for strict pseudo-contractions in Hilbert spaces," Journal of Mathematical Analysis and Applications, vol. 329, no. 1, pp. 336-346, 2007.

[13] H.-K. Xu, "Iterative algorithms for nonlinear operators," Journal of the London Mathematical Society. Second Series, vol. 66, no. 1, pp. 240-256, 2002.

[14] Y. Yao, Y.-C. Liou, and S. M. Kang, "Approach to common elements of variational inequality problems and fixed point problems via a relaxed extragradient method," Computers \& Mathematics with Applications, vol. 59, no. 11, pp. 3472-3480, 2010.

[15] J. Reinermann, "Über Fixpunkte kontrahierender Abbildungen und schwach konvergente Toeplitz-Verfahren," Archiv der Mathematik, vol. 20, pp. 59-64, 1969.

[16] F. Cianciaruso, G. Marino, L. Muglia, and Y. Yao, "A hybrid projection algorithm for finding solutions of mixed equilibrium problem and variational inequality problem," Fixed Point Theory and Applications, vol. 2010, Article ID 383740, 19 pages, 2010.

[17] G. Marino, L. Muglia, and Y. Yao, "Viscosity methods for common solutions of equilibrium and variational inequality problems via multi-step iterative algorithms and common fixed points," Nonlinear Analysis. Theory, Methods \& Applications, vol. 75, no. 4, pp. 1787-1798, 2012.

[18] L. C. Ceng, A. Petruşel, and J. C. Yao, "Relaxed extragradient methods with regularization for general system of variational inequalities with constraints of split feasibility and fixed point problems," Abstract and Applied Analysis, vol. 2013, Article ID 891232, 25 pages, 2013.

[19] W. Takahashi and M. Toyoda, "Weak convergence theorems for nonexpansive mappings and monotone mappings," Journal of Optimization Theory and Applications, vol. 118, no. 2, pp. 417428, 2003.

[20] A. Moudafi and P.-E. Maingé, "Towards viscosity approximations of hierarchical fixed-point problems," Fixed Point Theory and Applications, vol. 2006, Article ID 95453, 10 pages, 2006.

[21] P.-E. Maingé and A. Moudafi, "Strong convergence of an iterative method for hierarchical fixed-point problems," Pacific Journal of Optimization, vol. 3, no. 3, pp. 529-538, 2007. 


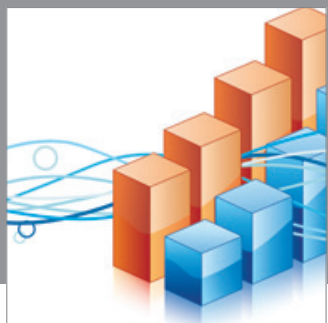

Advances in

Operations Research

mansans

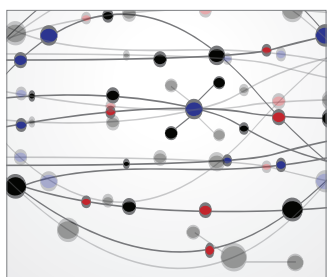

The Scientific World Journal
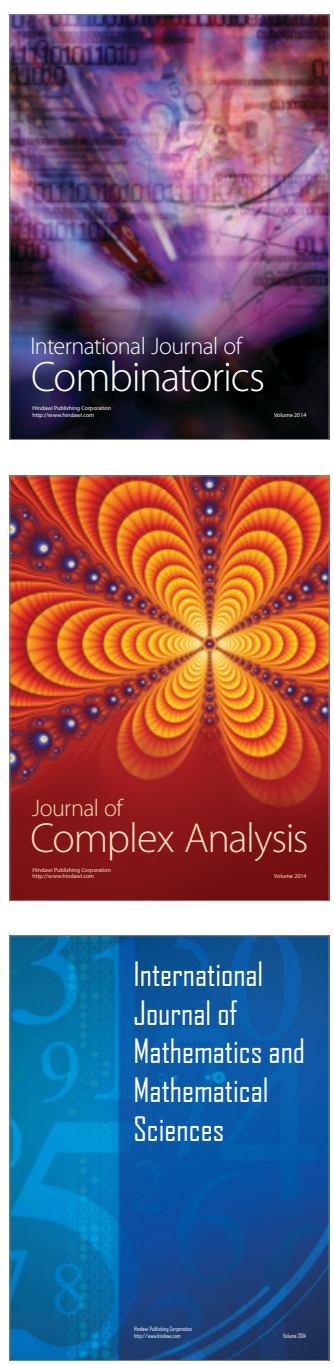
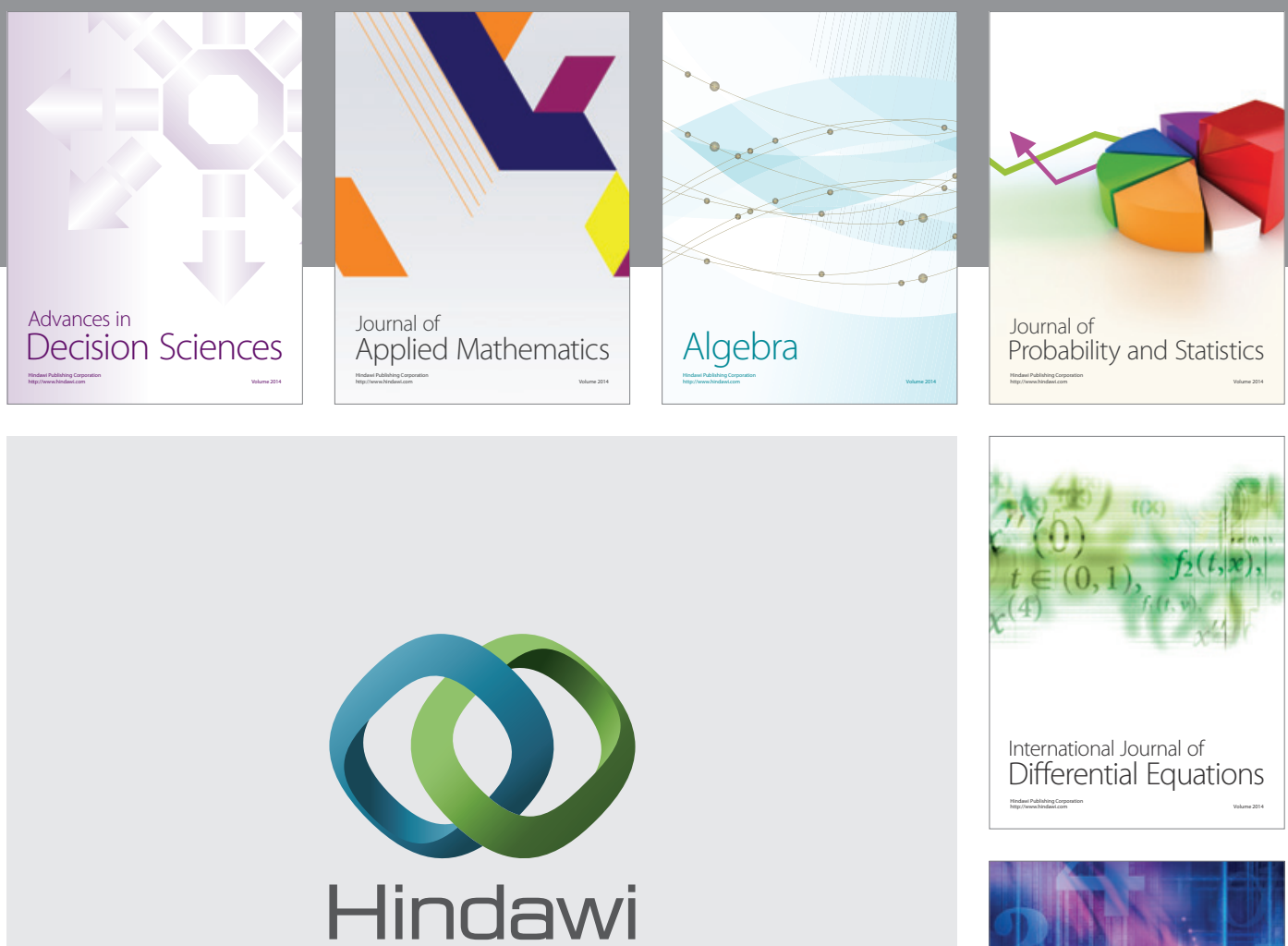

Submit your manuscripts at http://www.hindawi.com
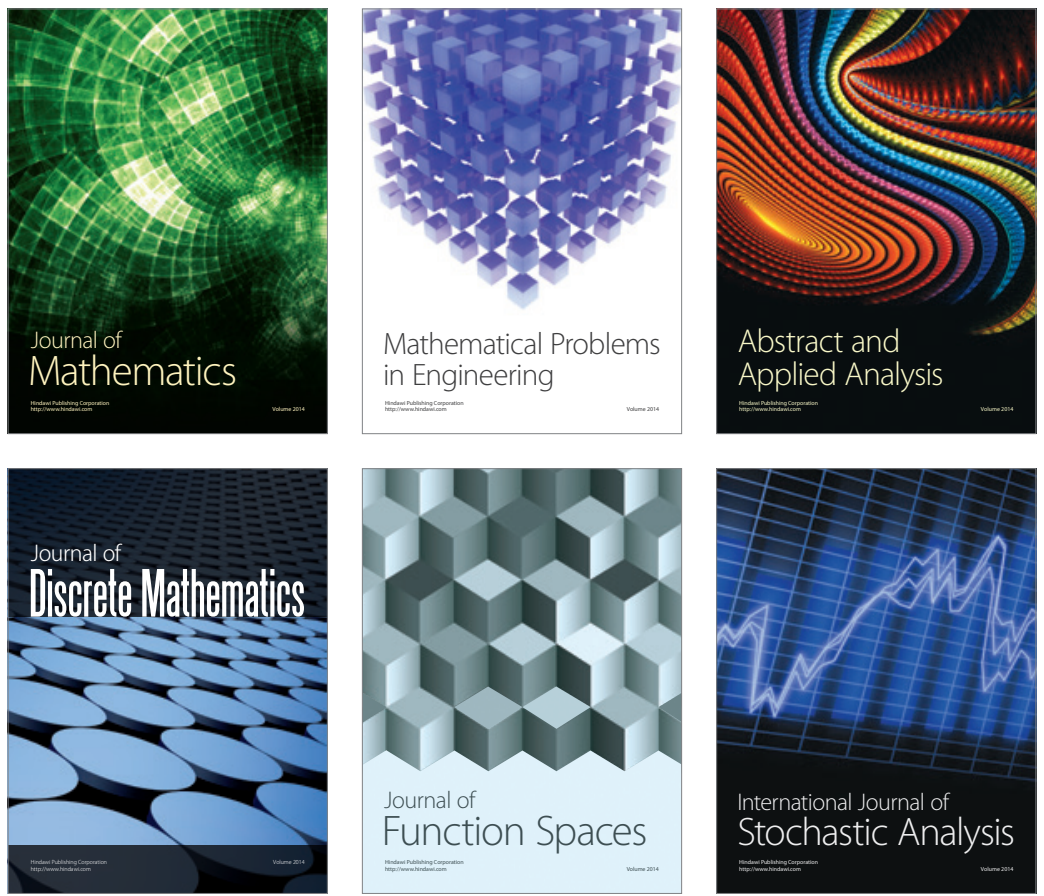

Journal of

Function Spaces

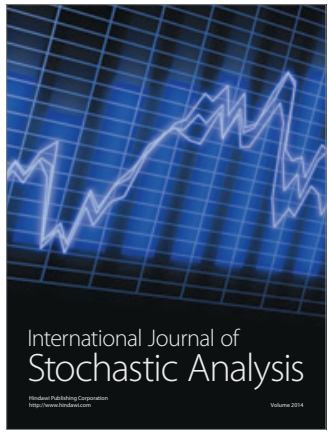

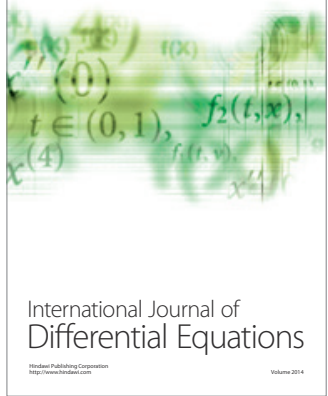
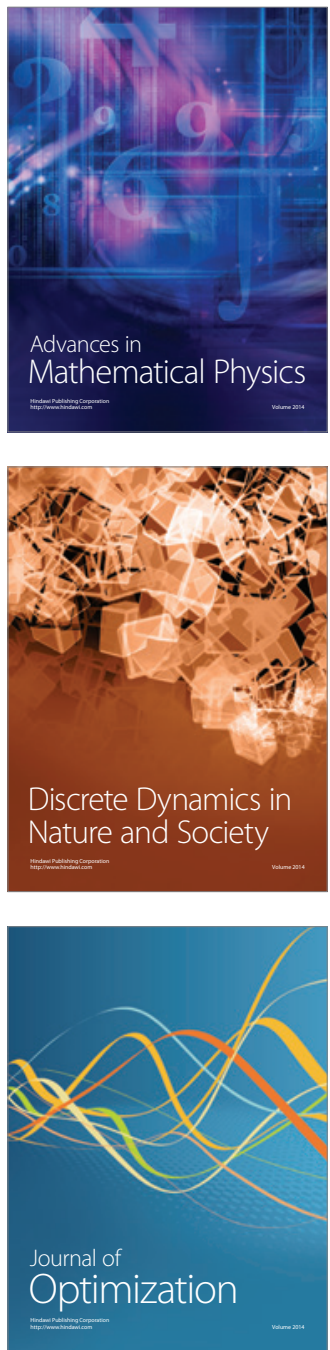